\title{
J-domain proteins promote client relay from Hsp70 during tail-anchored membrane protein targeting
}

Received for publication, January 22, 2021, and in revised form, February 22, 2021 Published, Papers in Press, March 17, 2021,

https://doi.org/10.1016/j.jbc.2021.100546

\author{
Hyunju Cho®, Woo Jun Shim ${ }^{\circ}$, Yumeng Liu, and Shu-ou Shan*(i) \\ From the Division of Chemistry and Chemical Engineering, California Institute of Technology, Pasadena, California, USA
}

Edited by Ursula Jakob

J-domain proteins (JDPs) play essential roles in Hsp70 function by assisting $\mathrm{Hsp70}$ in client trapping and regulating the Hsp70 ATPase cycle. Here, we report that JDPs can further enhance the targeting competence of Hsp70-bound client proteins during tail-anchored protein (TA) biogenesis. In the guided-entry-of-tail-anchored protein pathway in yeast, nascent TAs are captured by cytosolic Hsp70 and sequentially relayed to downstream chaperones, Sgt2 and Get3, for delivery to the ER. We found that two JDPs, Ydj1 and Sis1, function in parallel to support TA targeting to the ER in vivo. Biochemical analyses showed that, while Ydj1 and Sis1 differ in their ability to assist Hsp70 in TA trapping, both JDPs enhance the transfer of Hsp70-bound TAs to Sgt2. The ability of the JDPs to regulate the ATPase cycle of Hsp70 is essential for enhancing the transfer competence of Hsp70-bound TAs in vitro and for supporting TA insertion in vivo. These results demonstrate a role of JDPs in regulating the conformation of Hsp70-bound clients during membrane protein biogenesis.

The Hsp70 family comprises a central hub of the chaperone network that maintains cellular protein homeostasis and functions in every stage of the protein life cycle, from de novo folding, protein transport, to aggregate remodeling and degradation (1-3). The function of Hsp70s is governed by the ATPase cycle in its nucleotide binding domain (NBD), which is tightly coupled to interactions with cochaperones. In the ATPbound state, the $\alpha$-helical lid of the Hsp70 substrate binding domain (SBD) docks against the NBD and is away from the peptide binding site in the SBD, allowing rapid client binding and dissociation (4). Client proteins and a class of cochaperones, termed Hsp40 or J-domain proteins (JDPs), stimulate ATP hydrolysis on Hsp70, converting Hsp70 to the ADP state that binds client proteins with higher affinity and kinetic stability (1-4). Studies with peptide substrates suggested that this client trapping is due in part to the closing of the $\alpha$-helical lid over the peptide binding site in the SBD, although closing appears less pronounced with protein substrates $(5,6)$. Another class of cochaperones, the nucleotide exchange factors, facilitate client dissociation from Hsp70 by accelerating ADP release and, in some cases, directly contacting the Hsp70SBD to drive client displacement (7-9).

* For correspondence: Shu-ou Shan, sshan@caltech.edu.
JDPs have been reported to assist in Hsp70 function via two mechanisms that are mediated by distinct structural and functional domains. All JDPs contain a $\sim 70$ residue J-domain that binds ATP-bound Hsp70 at the NBD-SBD interface and stimulates their ATPase activity. A conserved His-Pro-Asp (HPD) motif in the J-domain docks at the interdomain linker of Hsp70 to optimize two networks of hydrogen bonding and hydrophobic interactions at the ATPase active site (10) and is essential for stimulating ATP hydrolysis on $\operatorname{Hsp70}(11,12)$. In addition, two substrate-binding C-terminal domains (CTDI/ CTDII) provide additional client interaction sites (13, 14). Although the role of the JDP/Hsp70 chaperone cycle in protecting and capturing client proteins is well established, whether their coupled chaperone cycle further modulates client conformation to promote folding has been a longstanding question. Recent works begin to address this question, showing that the bacterial JDP/Hsp70 homolog, DnaJ/K, can release luciferase in an altered conformation that folds more rapidly compared with that during spontaneous folding in dilute solution (15). Nevertheless, the generality of this phenomenon and whether JDPs participate in this client conformational modulation remain to be determined.

JDPs are divided into three classes (A, B, and C) based on the location of the J-domain and the presence of a zinc-finger-like region (16-18). Class A JDPs, such as Escherichia coli DnaJ and yeast Ydj1, contain an N-terminal J-domain, a glycine/ phenylalanine-rich linker, CTDI/CTDII that provide client binding sites, and a C-terminal dimerization domain. Class $\mathrm{B}$ JDPs, represented by yeast Sis1, share the same domain organization as Class A JDPs, except that they lack the zinc-fingerlike region insertion in the CTD-I of Class A JDPs. Class C JDPs contain additional specialized domains involved in diverse functions such as protein translation, pre-mRNA splicing, and clathrin-binding (19-21). Emerging evidence implicated all three classes of JDPs in an essential cellular process, the targeted delivery of nascent proteins to biological membranes $(22,23)$. The most abundant cytosolic JDPs, Ydj1 and Sis1, are required for the targeting of secretory proteins to the ER and the import of mitochondrial $\beta$-barrel and matrix proteins $(24,25)$. Ydj1 can bind a short cyclic $\beta$-hairpin on newly synthesized Tom40, suggesting that it plays a direct role in substrate recognition during targeting (25). Two additional cytosolic JDPs, Apj1 (Class A) and Jjj3 (Class C), were implicated in the targeting of glycosylphosphatidylinositol-anchored proteins to the ER (26). 
Finally, Xdj1 (Class A) and Djp1 (Class B) are required for the efficient assembly of the translocase of the outer mitochondrial membrane (27) and the biogenesis of the mitochondrial outermembrane protein Mim1 (28), respectively. Despite the abundance of in vivo data, the mechanism by which cytosolic JDPs participate in the biogenesis of membrane and organellar proteins remains unclear.

We address this question in the guided entry of tailanchored protein (GET) pathway, which delivers an essential class of tail-anchored membrane proteins (TAs) harboring hydrophobic transmembrane domains (TMDs) to the ER and is highly conserved across eukaryotic organisms. TAs contain a single TMD near the $C$ terminus and mediate diverse cellular processes such as protein translocation across organelle membranes, vesicular trafficking, protein quality control, and apoptosis $(29,30)$. Recently, we demonstrated that newly synthesized TAs released from the ribosome are captured by the yeast cytosolic Hsp70, Ssa1, which protects hydrophobic TMDs from aggregation in the cytosol and is crucial for maintaining TAs in a translocation-competent state (31). Ssa1 further initiates a cascade of energetically downhill TA transfer events, first to the downstream cochaperone Sgt2 and then the targeting factor Get3 (31), which delivers TAs to the Get1/2 receptor complex at the ER for insertion into the membrane. Although the role of Ssa1 in the GET pathway has been established (31), whether and how additional Ssa1 cochaperones participate in TA targeting is unknown. In this work, we show that Ydj1 and Sis1, representing two different classes of JDPs, function redundantly to support the targeting of GETdependent TAs in vivo. Unexpectedly, biochemical analyses showed that while Ydj1 assists Ssa1 in capturing TAs and preventing TA aggregation, Sis1 does not. On the other hand, both JDPs enhance the subsequent transfer of TA from Ssa1 to Sgt2. A functional J-domain in both JDPs was required to enhance the transfer competence of Ssa1-bound TA and to support efficient TA insertion in vivo. Our results uncover a new role of JDPs in regulating the conformation of Hsp70bound client proteins to enhance their targeting competence.

\section{Results}

\section{Ydj1 and Sis1 are essential for TA insertion into the ER in vivo}

We first asked whether cytosolic JDPs are involved in the ER targeting of TAs. Because two JDPs, Ydj1 and Sis1, were implicated in the translocation of $\alpha$ factor into the ER and the import of mitochondrial precursor proteins $(24,25)$, we hypothesized that they also assist Ssa1 in TA targeting to the ER membrane in vivo. To test this hypothesis, we used an established set of isogenic yeast strains in which the expression of YDJ1, SIS1, or both genes are under the control of a tetracycline-repressible promoter (tet-YDJ1, tet-SIS1, and tetYDJ1/tet-SIS1; (25)). We depleted Ydj1 and/or Sis1 in these strains using $4 \mathrm{~h}$ of doxycycline treatment (Fig. S1), as described (25), and carried out pulse-chase assays to measure the insertion kinetics of newly synthesized model substrates into the ER (Fig. 1A). We used BirA fused to the C-terminal TMD of the SNARE protein Bos1 (BirA-Bos1) as the model
TA for the GET pathway, because it is strongly dependent on Ssa1 and Get3 for insertion into the ER (31). Successful insertion leads to efficient glycosylation of an opsin tag at the C terminus of BirA-Bos1 (Cho and Shan, 2018), providing a quantitative readout for targeting and translocation efficiency. We found that depletion of either Ydj1 or Sis1 did not significantly affect the insertion efficiency of BirA-Bos1 (Fig. 1, $B$ and $C$ ), whereas the depletion of both nearly abolished BirABos1 insertion (Fig. 1D).

To exclude the possibility that the observed translocation defect of BirA-Bos1 arose from pleiotropic effects in tet-YDJ1/ tet-SIS1 cells, such as a reduced cytosolic folding capacity or compromised ER membrane, we tested two control substrates, Bos1-BirA and DHC- $\alpha \mathrm{F}$, whose targeting and translocation are independent of Hsp70 and Get3 (Fig. 1A) (31). In Bos1-BirA, the Bos1-TMD is upstream of the BirA moiety and near the $\mathrm{N}$ terminus of the protein, making it a substrate for the cotranslational signal recognition particle (SRP) pathway (31). In DHC- $\alpha \mathrm{F}$, the $\mathrm{N}$-terminal signal sequence of prepro- $\alpha$ factor is replaced by the TMD of aminopeptidase B (DAP2) to convert it into an SRP-dependent substrate that uses the cotranslational targeting pathway (32). Pulse-chase experiments showed that depletion of both Ydj1 and Sis1 had only modest effects on the translocation of Bos1-BirA (Fig. 1, E-G) and DHC- $\alpha$ F (Fig. $1, H-J$ ), suggesting that nonspecific folding defects are insufficient to account for the large deleterious effects of the Ydj1/Sis1 depletion on the ER insertion of BirA-Bos1.

It has been reported that yeast harbors multiple alternative targeting pathways to mediate post-translational membrane protein targeting to the ER (33). For example, the SRPindependent (SND) pathway mediates the targeting of proteins with an internal TMD (34), and TAs with low hydrophobicity TMDs are targeted by GET-independent pathways $(35,36)$. To test whether the Hsp70/JDP system functions in these alternative pathways, we used two established substrates, BirA-6AG (35) and Scs2-GFP (34) (Fig. S2A). BirA-6AG is a GET-independent TA in which multiple hydrophobic residues in the Bos1 TMD are replaced by Ala/Gly to reduce hydrophobicity (Fig. S2B) (35). Scs2-GFP is an established SND substrate containing an internal TMD (34). We tested the Ydj1/Sis1-dependence for the translocation of both substrates using the tet-YDJ1/tet-SIS1 cells as above. We also tested the Hsp70-dependence using the established SSA1 (SSA1ssa2Assa $3 \Delta s s a 4 \Delta)$ and $s s a 1^{t s}\left(s s a 1^{t s} s s a 2 \Delta s s a 3 \Delta s s a 4 \Delta\right)$ strains, in which a temperature-sensitive mutant Ssal in $s s a 1^{t s}$ is rapidly inactivated within $5 \mathrm{~min}$ upon shift to nonpermissive temperature (37). While the ER targeting of both BirA-6AG and Scs2-GFP proteins are independent of Get3 (Fig. S2C), the translocation efficiencies of both substrates were reduced substantially upon transient inactivation of Ssa1 or depletion of Ydj1/Sis1 (Fig. S2, D-G). These results strongly suggest that the cytosolic Hsp70/JDP system, comprised of Ydj1/Sis1 and Ssa1, is involved in the post-translational ER targeting of multiple classes of membrane proteins in yeast.

Together, these results show that two cytosolic JDPs, Ydj1 and Sis1, function redundantly to facilitate the post-translational targeting of TAs to the ER in vivo. The strong TA 
A

BirA-Bos1

\begin{tabular}{|l|l|l|l|}
\hline $3 x H A$ & BirA & Bos1-TMD & opsin \\
\hline
\end{tabular}

B

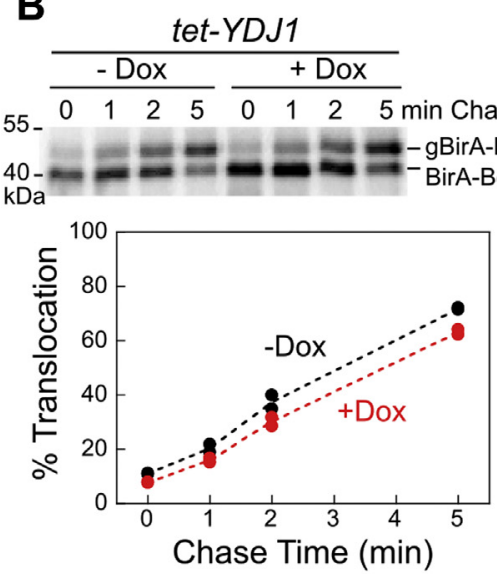

E
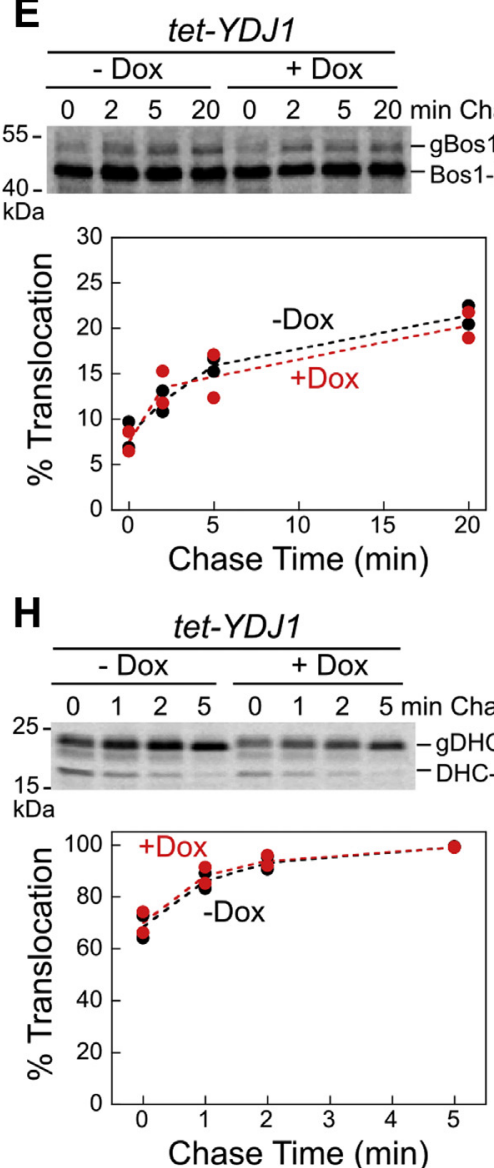

Bos1-BirA

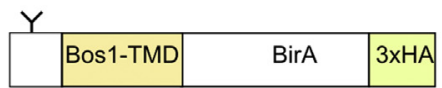

C

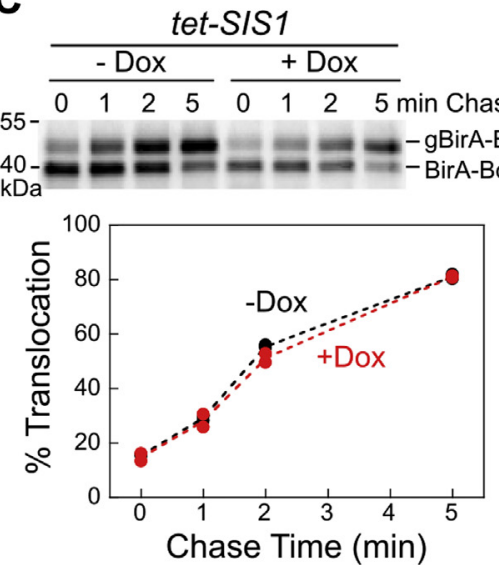

$\mathbf{F}$

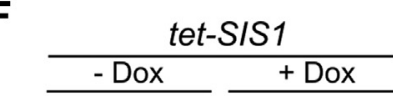
$\begin{array}{llllllll}0 & 2 & 5 & 20 & 0 & 2 & 5 & 20 \\ \text { min Chase }\end{array}$

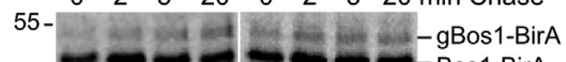
$40-$
$\mathrm{kDa}$

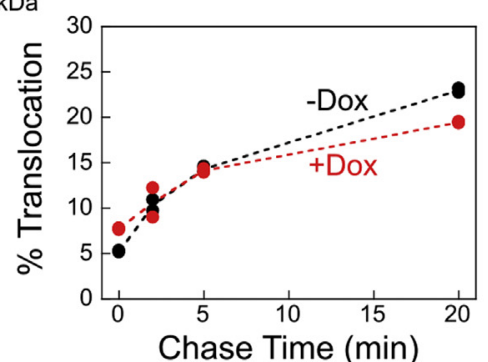

I

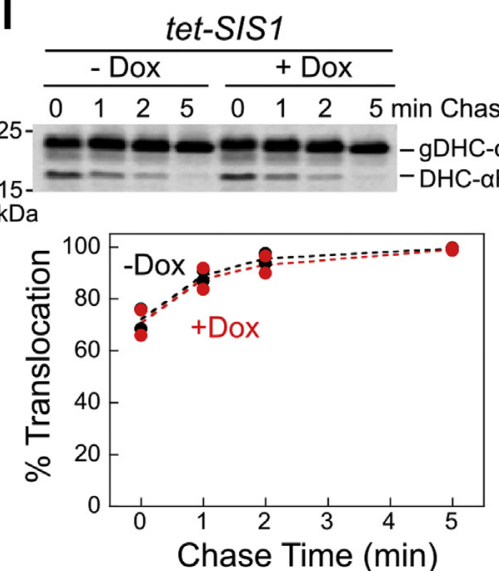

DHC- $\alpha \mathrm{F}$

\begin{tabular}{|c|c|c|}
\multicolumn{3}{|c}{$Y Y$} \\
\hline DAP2-TMD & aF & $3 \times H A$ \\
\hline
\end{tabular}

D

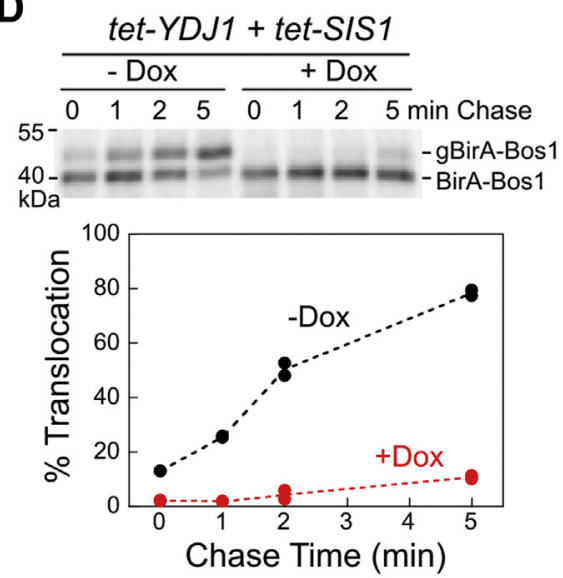

G
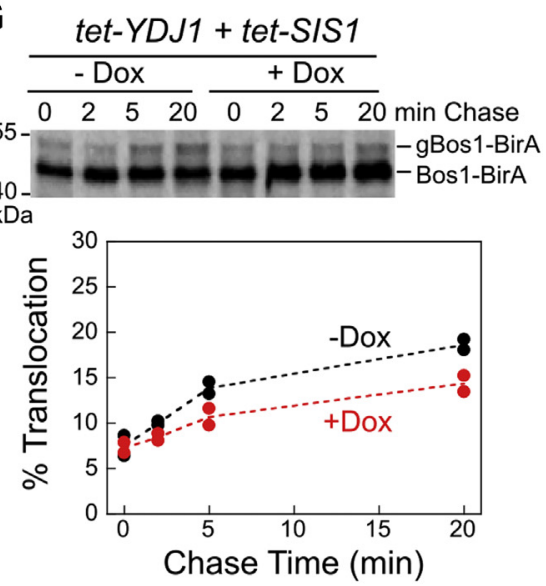

J

Jet-YDJ1+tet-SIS1
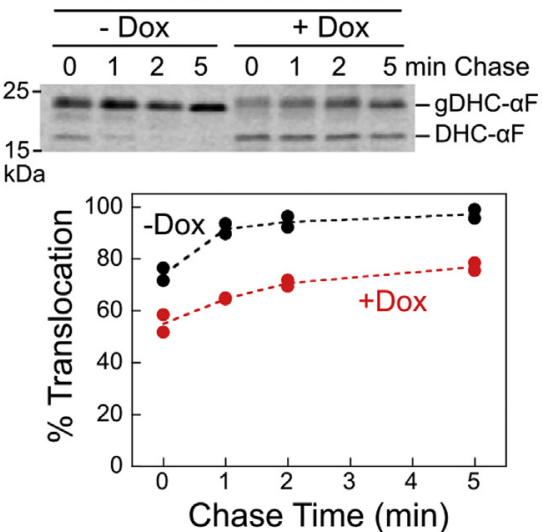

Figure 1. Depletion of both Ydj1 and Sis1 abolish the ER targeting of TAs in vivo. A, scheme of model substrates used in the in vivo targeting assay (31). " $\mathrm{Y}$ " denotes the glycosylation sites that allow detection of substrate insertion into the ER in the correct topology. $\mathrm{N}$ - or C-terminal 3xHA tags were used for immunoprecipitation of the substrates. $B-J$, top, representative autoradiograms for pulse-chase analysis of the translocation of metabolically labeled BirABos1 $(B-D)$, Bos1-BirA $(E-G)$, and DHC-aF $(H-J)$ in tet-YDJ1, tet-SIS1, and tet-YDJ1/tet-SIS1 cells in the absence $(-)$ and presence (+) of Doxycycline (Dox). " $\mathrm{g}$ " denotes glycosylated substrates, which migrate more slowly than nonglycosylated substrates. Bottom, quantification of the data in $(B-J)$ and their replicates. Values from two biological replicates are shown as black circles (-Dox) and red circles (+Dox). TA, tail-anchored protein. 
insertion defect upon depletion of both JDPs further indicate that at least one of them is required for the efficient targeting of GET-dependent substrates and that this role cannot be filled by other JDPs. Finally, this JDP/Hsp70 system is also required for the efficient targeting of GET-independent TAs and SND substrates, suggesting that they form a chaperone hub upstream of diverse post-translational membrane protein targeting pathways in yeast.

\section{Ydj1 and Sis 1 differ in their ability to assist Hsp70 in substrate trapping}

We investigated the molecular mechanisms by which Ydj1 and Sis1 facilitate the ER targeting of TAs in biochemical analyses. As Ssa1 is required to capture nascent TAs in the soluble form (31), and as JDPs have been suggested to autonomously bind substrates and to assist Hsp70s in substrate trapping (10, 14, 38, 39), we first asked whether Ydj1 and Sis1 enhance the ability of Ssa1 to capture TAs and prevent them from aggregation. As the model TA substrate in vitro, we used a noncleavable, soluble SUMO domain fused to the Bos1 TMD (termed Bos1) (31, 35, 40). Dilution of purified, detergent-solubilized Bos1 into aqueous buffer, which removes detergent micelles, led to rapid aggregation of Bos1 as monitored by the turbidity assay (Fig. 2A, light blue line). Using this assay, we confirmed that ATP-bound Ssa1 effectively prevented Bos1 aggregation (Figs. $\mathrm{S} 3 A$ and $\mathrm{S} 1 B$, red), as reported (31). In comparison, ADP-bound Ssa1 prevented
A

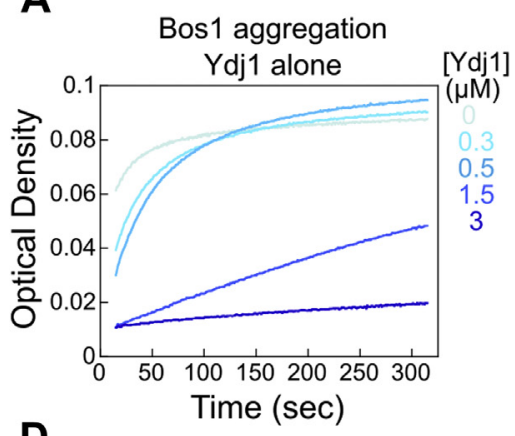

D
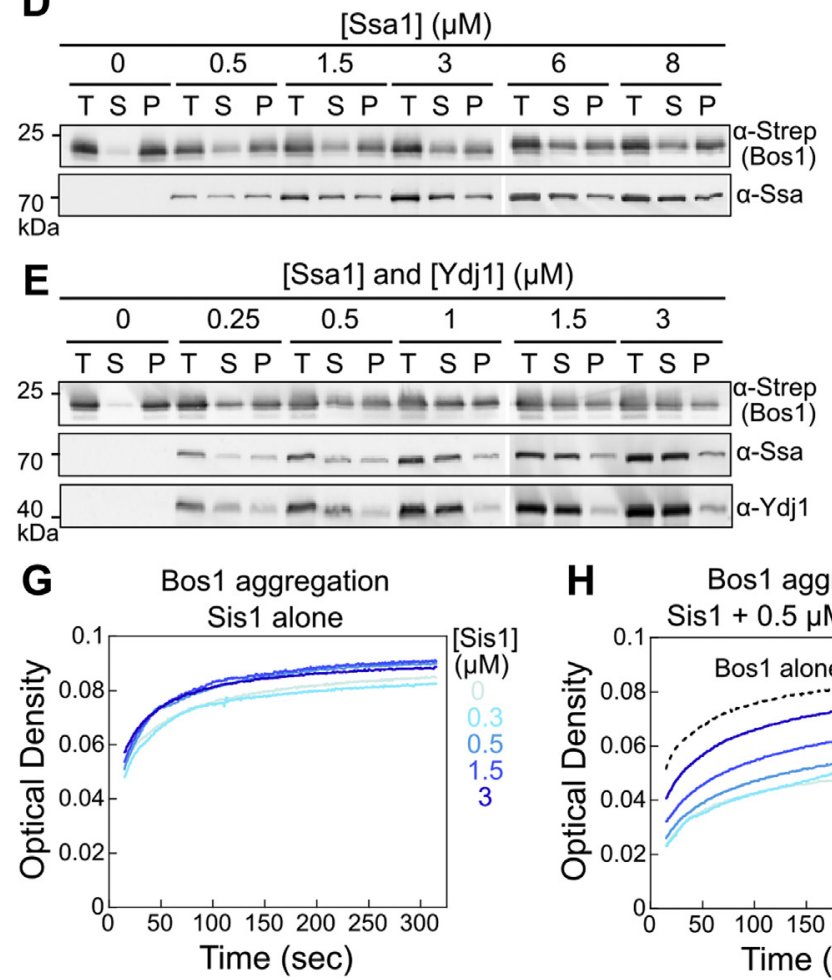

B

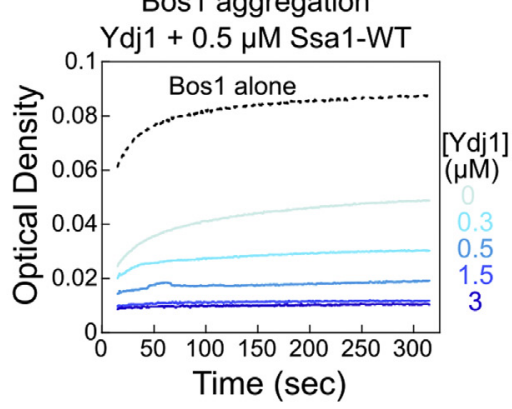

C

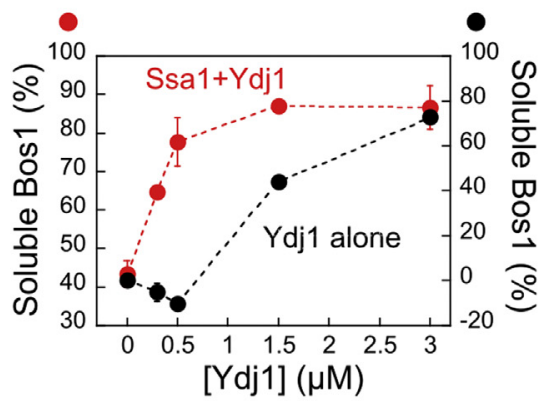

$\mathbf{F}$

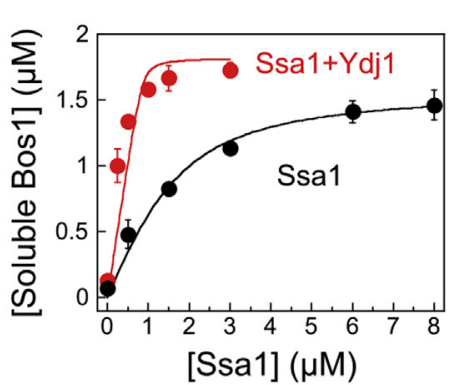

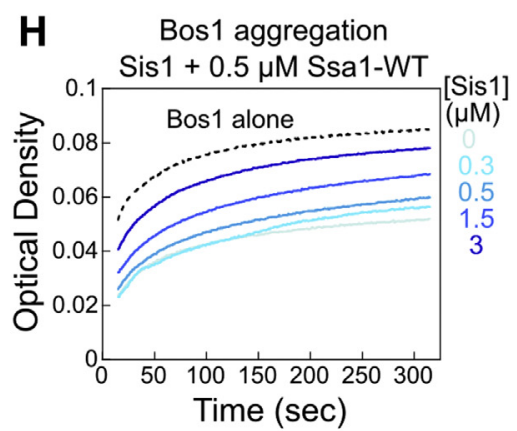

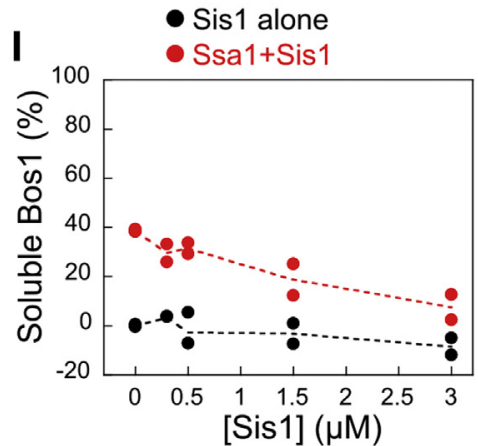

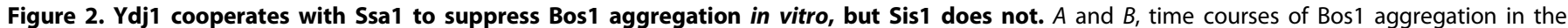

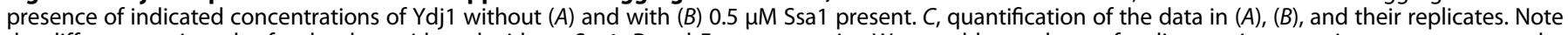

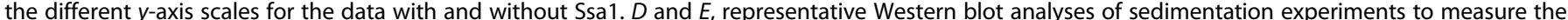

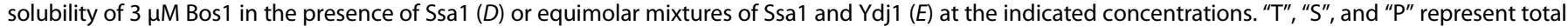

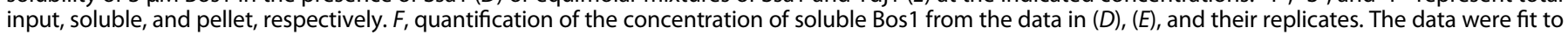

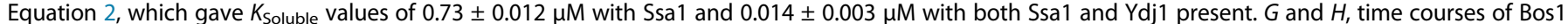

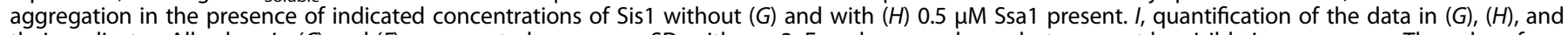

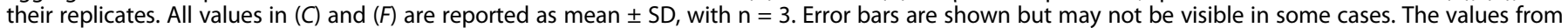
two independent experiments are shown in (I) as black (Ssa1 alone) and red (Ssa1+Sis1) circles. 
TA aggregation less efficiently (Fig. S3, $A$ and $B$, green versus red), and apo-Ssa1 was unable to suppress TA aggregation (Fig. S3, $A$ and $B, b l u e)$. These results show that nucleotide binding is required for Ssa1 to capture TAs in the soluble form, and that ATP-bound Ssa1 is most efficient in TA capture.
Ydj1 by itself only prevented Bos1 aggregation at superstoichiometric concentrations ( $\geq 3 \mu \mathrm{M}$ Ydj1 versus $1.5 \mu \mathrm{M}$ Bos1; Fig. 2A). In comparison, $0.5 \mu \mathrm{M}$ Ssa1 prevented the aggregation of $\sim 50 \%$ Bos1 (Fig. $2 B$, dotted black versus light blue lines), as reported (31), indicating that Ssa1 is a more
A

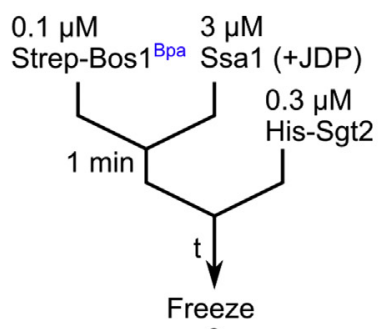

$\&$

UV crosslink at $-20^{\circ} \mathrm{C}$
B Bos $1^{\text {Bpa }}$ transfer from Ssa1 to Sgt2

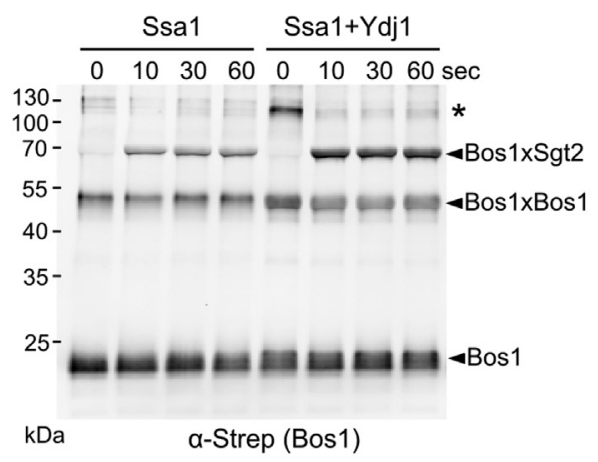

C

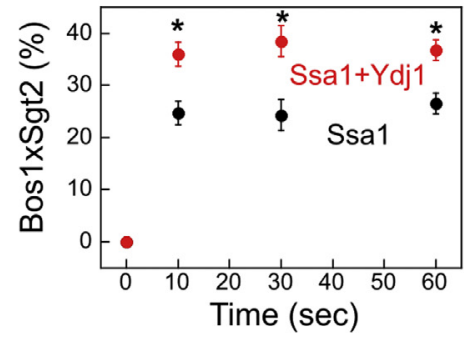

D

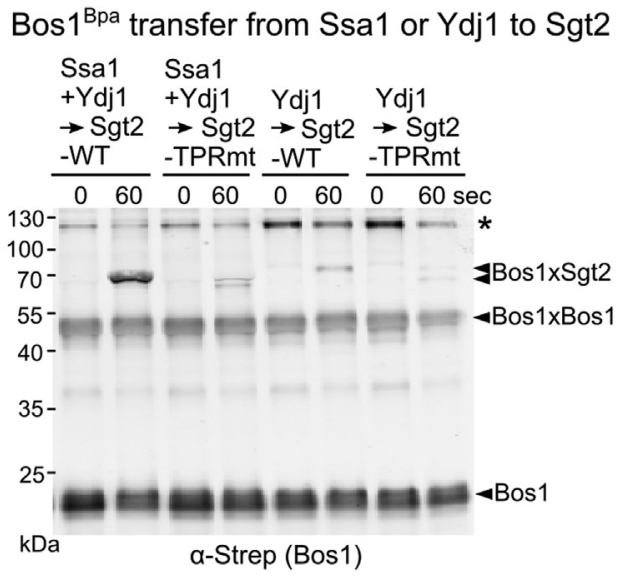

$\mathbf{F}$

Bos $1^{\text {Bpa }}$ transfer from Ssa1 to Sgt2

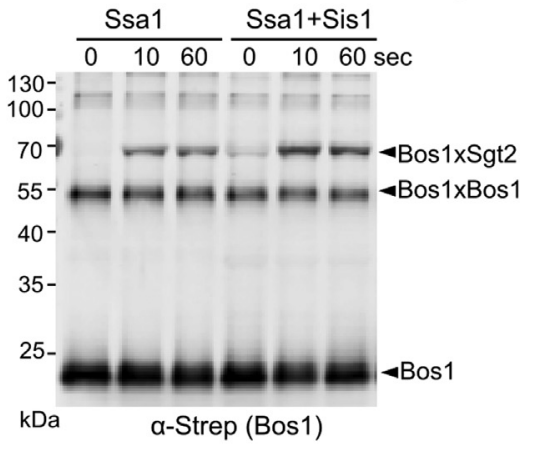

G

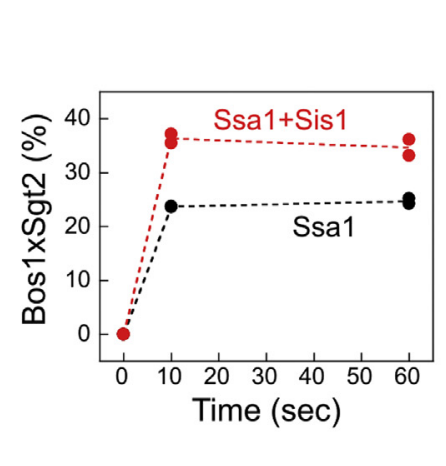

E

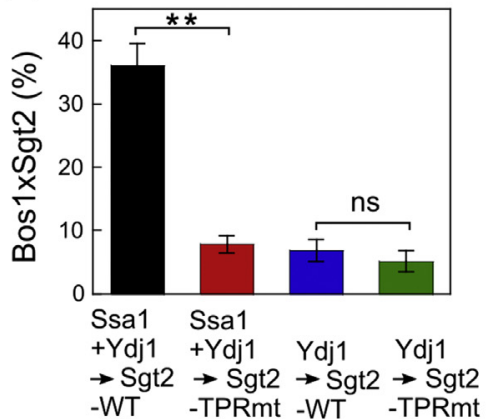

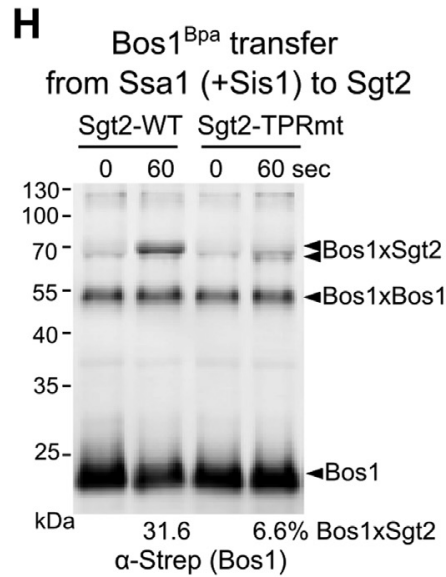

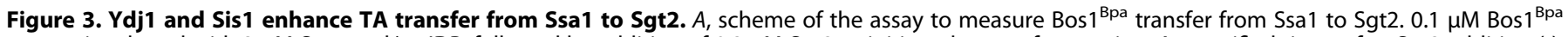

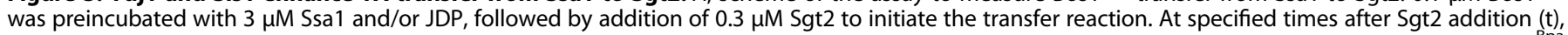

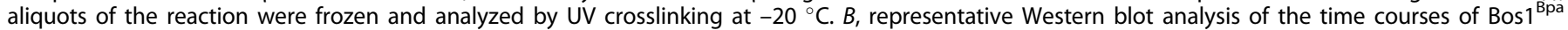

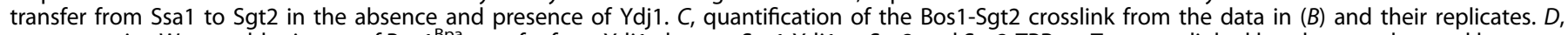

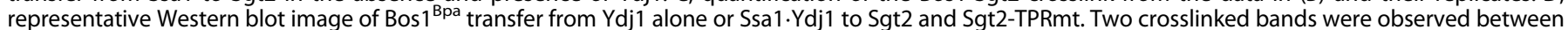

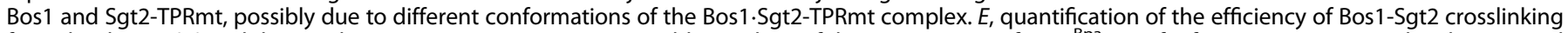

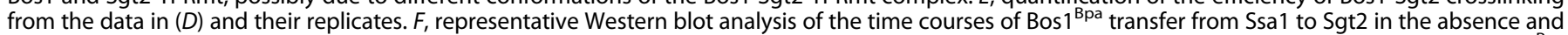

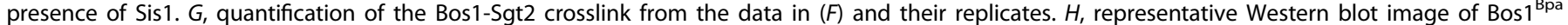

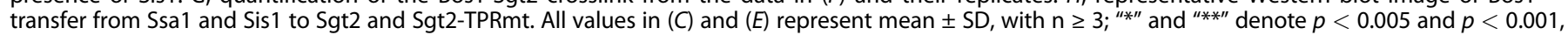

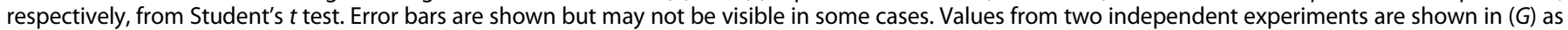
black (Ssa1 alone) and red (Ssa1+Sis1) circles. TA, tail-anchored protein. 
effective chaperone than Ydj1 for Bos1. In the presence of $0.5 \mu \mathrm{M}$ Ssa1, addition of substoichiometric amounts of Ydj1 significantly enhanced the solubility of Bos1 (Fig. 2, $B$ and $C$, red), suggesting that Ydj1 cooperates with Ssa1 to prevent Bos1 aggregation. To better estimate the efficacy of the Ydj1-Ssa1 complex in TA capture, we used a sedimentationbased assay that detects both the soluble (supernatant) and insoluble (pellet) fractions of Bos1 (Fig. 2, D and E). In the absence of Ydj1, Ssa1 generated a maximum of $1.5 \mu \mathrm{M}$ soluble Bos1 at saturating concentrations, and $>3 \mu \mathrm{M}$ Ssa1 was required for saturation (Fig. 2F, black circles). With equimolar Ydj1 and Ssa1 present, the maximum amount of soluble TA substrate was similar (1.7 versus $1.5 \mu \mathrm{M})$, but saturation occurred at lower Ssa1 concentrations (Fig. 2F, red circles). Analysis of the chaperone concentration dependences of the sedimentation data yielded apparent constants $\left(K_{\text {soluble }}\right)$ of $0.014 \pm 0.003$ and $0.73 \pm 0.012 \mu \mathrm{M}$, respectively, for the chaperoning of Bos1 by Ssa1 with and without Ydj1. These results provide independent evidence for the synergy between Ydj1 and Ssa1 in the capture and maintenance of soluble TA.

In contrast to Ydj1, Sis1 displayed no autonomous chaperone activity toward Bos1 (Fig. 2G). In the presence of $0.5 \mu \mathrm{M}$ Ssa1, the addition of Sis1 reduced rather than further improved the solubility of Bos1 (Fig. 2, $H$ and I). These observations are consistent with the previously reported difference in the client binding domains and properties of Ydj1 and Sis1 $(41,42)$. Importantly, the inability of Sis1 to chaperone TAs either by itself or in concert with Ssa1 strongly suggests that these activities are not required for its role in facilitating TA insertion into the ER in vivo (Fig. 1). Thus, the roles of JDPs in supporting TA insertion are unlikely to be solely attributed to enhanced TA capture by the Ssa1/JDP pair.

\section{Ydj1 and Sis1 enhance TA transfer from Ssa1 to Sgt2}

The only other major step involving Ssa1 in the GET pathway is the transfer of its bound TA to the cochaperone Sgt2 (31). To test if the JDPs regulate this step, we monitored TA transfer using a photocrosslinker, p-benzoyl-l-phenylalanine (Bpa), site-specifically incorporated into the eighth residue (Ile) in the Bos1-TMD using amber suppression (43). Purified, Bpa-incorporated Bos1 $\left(\mathrm{Bos} 1^{\mathrm{Bpa}}\right.$ ) was preincubated with Ssa1 for $1 \mathrm{~min}$ to form the soluble Ssa1-Bos $1^{\text {Bpa }}$ complex, followed by the addition of $\mathrm{His}_{6}$-tagged Sgt 2 to initiate TA transfer (Fig. 3A). Before and at different times during the transfer, aliquots of the reaction were flash frozen, and the chaperone association of $\operatorname{Bos} 1^{\mathrm{Bpa}}$ was monitored by UVinduced photocrosslinking (Figs. $3 A$ and S4). Both Ssa1 and Ydj1 form $\sim 125 \mathrm{kDa}$ crosslinks to Bos1 ${ }^{\text {Bpa }}$ (Fig. S4, $A-E$ ). Compared with the Bos1-Ydj1 crosslink, the Bos1-Ssa1 crosslink was weak and diffuse (Fig. S4, $A-E$ ) despite the more effective TA capture by Ssa1 than Ydj1 (Fig. 2 and (31)), likely reflecting the more dynamic nature of the TA interaction with Ssa1. In contrast, the Bos1-Sgt2 crosslink at $\sim 70 \mathrm{kDa}$ was distinct and readily detectable (Figs. $3 B$ and $\mathrm{S} 4$ ). We therefore monitored the efficiency of the Ssa1-to-Sgt2 TA transfer based on the Bos1-Sgt2 crosslink.
Upon the addition of Sgt2, the Bos1-Ssa1 crosslink at $\sim 125 \mathrm{kDa}$ disappeared and was replaced by a crosslinked band to Sgt2 (Fig. S4, B and C), indicating the transfer of TA from Ssa1 to Sgt2. Consistent with the results of fluorescence-based TA transfer assays (31), the Bos1-Sgt2 crosslink was substantially reduced by the R171A, R175A mutations on the conserved tetratricopeptide repeat (TPR) domain of Sgt2 (TPRmt), which disrupts Sgt2 binding with Ssa1 (40), corroborating that the transfer was strongly dependent on the Ssa1-Sgt2 interaction (Figs. 3C and S5, $A-C$; (31)).

We asked if Ydj1 participates in the TA transfer event. Time course measurements showed that TA transfer from Ssa1 to Sgt 2 was rapid and complete within $10 \mathrm{~s}$ both with and without Ydj1 present (Figs. $3 B$ and S5). Compared to the Ssa1-Bos1 complex formed without Ydj1, the presence of Ydj1 during the preincubation of Ssa1 with TA significantly increased the fraction of TA substrate crosslinked to Sgt2 $(36.8 \pm 1.1 \%$ versus $26.5 \pm 2.2 \%)$ (Fig. $3, B$ and $C)$. We excluded the possibility that this enhancement was due to the presence of an additional pathway in which Ydj1 directly transfers its bound TA to Sgt2. First, the transfer reaction with both Ssa1 and Ydj1 present is abolished by the R171A, R175A mutations on the conserved TPR domain of Sgt2 (TPRmt) that disrupt its binding with Ssa1 (40) (Fig. 3, $D$ and $E$ ). Thus, the transfer reaction in the presence of Ydj1 is still dependent on the interaction of Ssa1 with the Sgt2 TPR domain, as was expected for a direct Ssa1-to-Sgt2 TA transfer (Fig. S5, $A-C$; (31)). In addition, only low levels of Bos1-Sgt2 crosslink were detected when Bos1 premixed with Ydj1 was incubated with Sgt2, and the Sgt2(TPRmt) did not affect direct TA transfer from Ydj1 to Sgt2 (Fig. 3, $D$ and E, blue and green). Thus, even in the presence of Ydj1, the Ssa1-to-Sgt2 transfer is the dominant pathway for TA loading on Sgt2, and this transfer was enhanced by Ydj1.

The following observations indicate that this enhancement is distinct from the roles of Ydj1 in assisting the capture of soluble TA. First, a saturating Ssa1 concentration $(3 \mu \mathrm{M})$ was used during the preincubation with TA before initiation of transfer, bypassing the Ydj1-mediated enhancement of TA binding by Ssa1. Second, when we changed the order of addition and supplemented Ydj1 to Sgt2 during the transfer phase after Bos1 was preloaded on Ssa1, the enhancement in the Bos1-Sgt2 crosslink persisted (Fig. S6). This ruled out models in which a pool of TA substrates was irreversibly trapped in transfer-incompetent conformations if the initial TA capture occurred without Ydj1. Third, as shown in the next section, the effects of Ydj1 on the TA capture and transfer steps have distinct molecular requirements and can be experimentally uncoupled. Finally, the same enhancement in Ssa1-to-Sgt2 TA transfer was observed with Sis1. Despite the inability of Sis1 to chaperone the TA substrate or to assist Ssa1 in TA trapping (Fig. 2, $H$ and $I$ ), the presence of Sis1 significantly increased the fraction of Bos $1^{\mathrm{Bpa}}$ crosslinked to Sgt2 after the transfer (Fig. 3, F and G), with efficiency comparable to that in the 
presence of Ydj1. The enhanced TA transfer from Ssa1-Sis1 to Sgt2 is also dependent on the interaction of Ssa1 with the Sgt2 TPR domain (Fig. $3 H$ ), supporting a direct TA transfer from Ssa1 to Sgt2 in the presence of Sis1. Together, these data strongly suggest an additional regulatory role of JDPs in enhancing the transfer competence of Ssa1-bound TA to Sgt2, thus committing the TA to localization at the ER membrane.
A

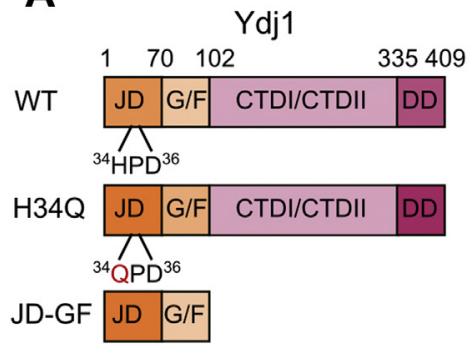

B

Sis1

WT \begin{tabular}{|l|l|l|l|l|l|}
1 & 68 & 121 & 172 & 336352 \\
\hline JD & G/F & $\mathrm{G} / \mathrm{M}$ & $\mathrm{CTDI} / \mathrm{CTDII}$ & $\mathrm{DD}$ \\
\hline
\end{tabular}

\begin{tabular}{l|l|l|l|l|l|} 
H34Q JD & G/F & G/M & CTDI/CTDII & $D D$ \\
\hline
\end{tabular} ${ }^{34} \mathrm{QPD}^{36}$

$3 K N \quad$\begin{tabular}{|l|l|l|l|l|}
\hline JD & G/F & G/M & CTDI/CTDII & $D D$ \\
\hline & $\prod_{199} \mathrm{~N}^{202} \mathrm{~N}^{214} \mathrm{~N}$
\end{tabular}

JD-GF JD $G / F$

\section{C}

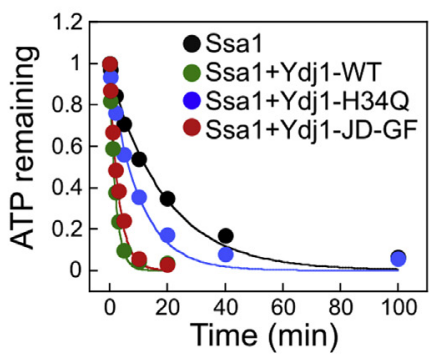

E

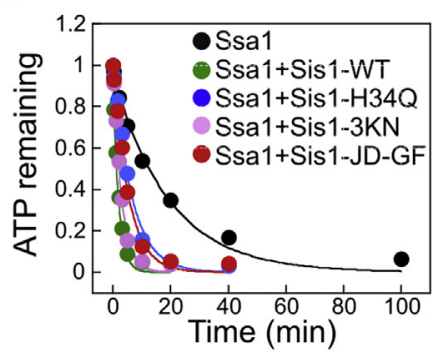

D

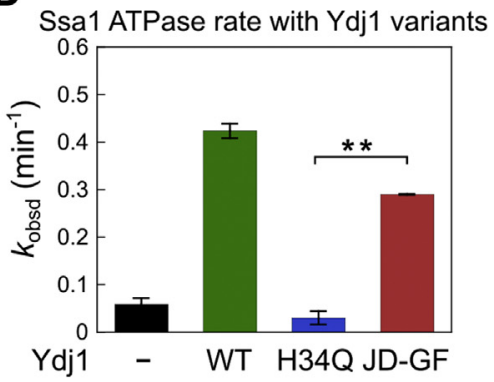

F Ssa1 ATPase rate with Sis1 variants

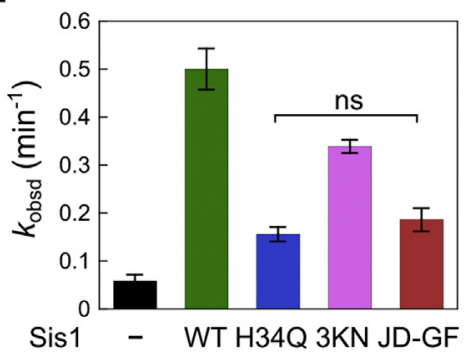

G Bos1 aggregation Ydj1 variants

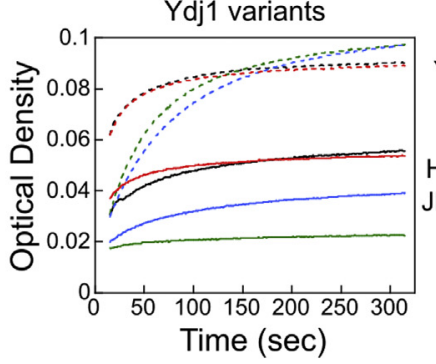

I

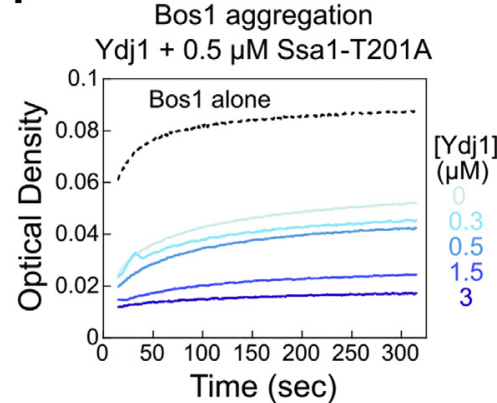

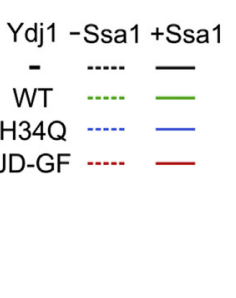

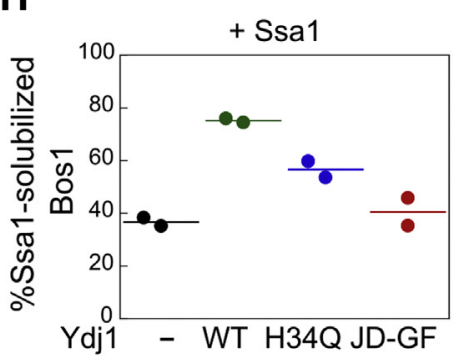

$\mathbf{J}$

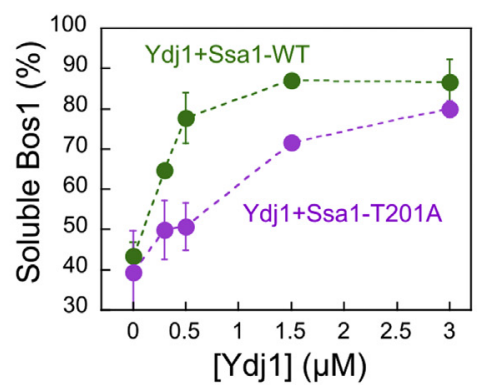

Figure 4. Ydj1 assists Ssa1 in TA capture in a soluble form using both its CTDs and J-domain. $A$ and $B$, schematic of the domain organization of wildtype and mutant Ydj1 $(A)$ and Sis1 $(B)$ tested in this work. $C-F$, representative time courses for single-turnover ATP hydrolysis reactions were shown for reactions with $3 \mu \mathrm{M}$ Ssa1 in the absence and presence of $3 \mu \mathrm{M}$ wildtype and mutant Ydj1 $(C)$ or Sis1 (E). ATPase reactions were measured and analyzed as described under Experimental procedures. Lines are fits of the data to Equation 3, and the obtained ATPase rate constants are summarized in parts $(D)$ and $(F)$. $G$, time courses of Bos 1 aggregation in the presence of $0.5 \mu \mathrm{M}$ of the indicated Ydj1 variants with (solid lines) and without (dotted lines) $0.5 \mu \mathrm{M}$ Ssa1. $\mathrm{H}$, the difference in optical density between the (-Ssa1) and (+Ssa1) reactions in $(G)$ and their replicates are quantified. The lines represent the mean from two independent experiments. I, time courses of Bos 1 aggregation in the presence of $0.5 \mu \mathrm{M}$ Ssa1(T201A) and indicated concentrations of Ydj1. J, quantification of the data in $(I)$ and their replicates (purple). The data for $0.5 \mu \mathrm{M}$ Ssa1-WT and Ydj1 (green) were from Figure $2 C$ and shown for comparison. All values in $(D)$, $(F)$, and $(J)$ are reported as mean $\pm S D$, with $n \geq 3$. " $* * * \prime$ denotes $p<0.001$ from Student's $t$ test. Error bars are shown but may not be visible in some cases. CTDI/CTDII, C-terminal domains I and II; DD, dimerization domain; G/F, Glycine and Phenylalanine-rich linker; G/M, Glycine and Methionine-rich region; JD, Jdomain; TA, tail-anchored protein. 


\section{A functional $\mathrm{J}$-domain is required to enhance the transfer competence of Ssa1-bound TA}

To decipher how JDPs cooperate with Ssa1 in the GET pathway, we tested mutations that block their distinct activities (Fig. 4, $A$ and $B$ ). The H34Q mutation in the conserved HPD motif of Ydj1 and Sis1 abolishes the ability of the J-domain to activate ATP hydrolysis in Hsp70 (Fig. 4, C-F) but does not affect client-binding in CTDI/CTDII $(11,13,39)$. JD-GF contains the JD and glycine/phenylalanine linker of Ydj1 or Sis1, which binds and regulates Hsp70, but lacks the CTDI/ CTDII that bind client proteins. Ydj1(JD-GF) activated the ATPase reaction of Ssa1 $\sim 5$-fold, only 30\% lower than

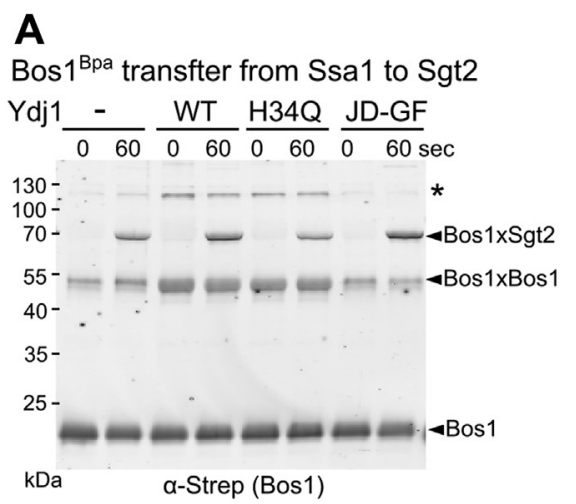

B

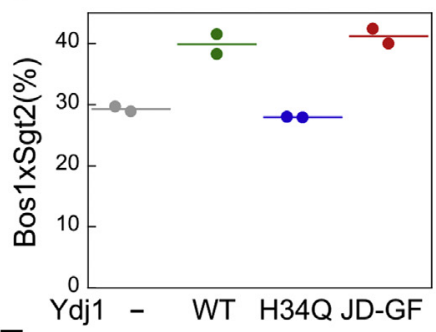

E

Bos $1^{\text {Bpa }}$ transfer from Ssa1-T201A to Sgt2

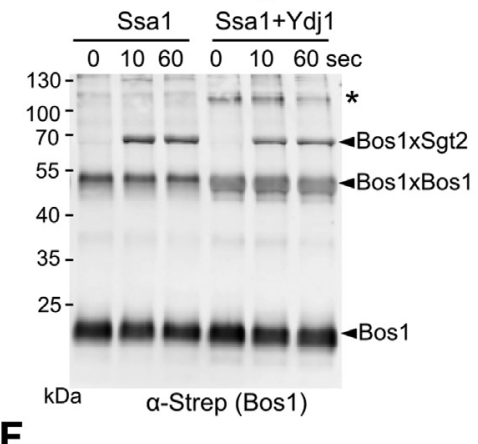

$\mathbf{F}$

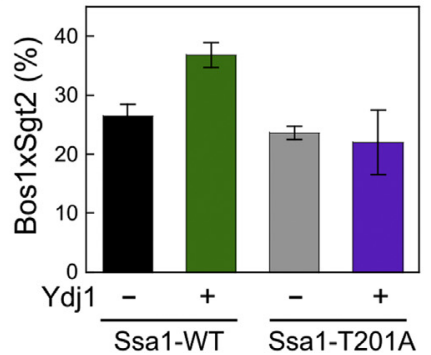

C Bos $1^{\text {Bpa }}$ transfter from Ssa1 to Sgt2

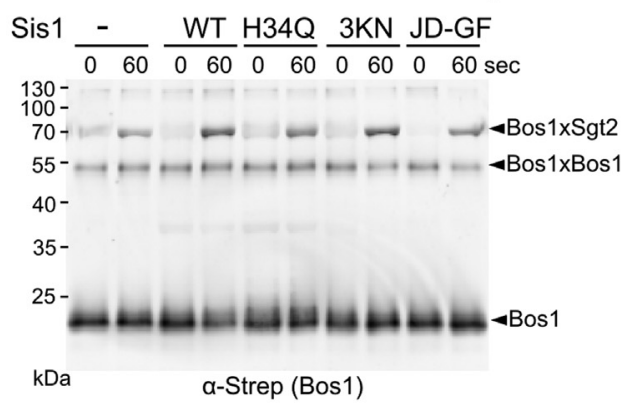

D

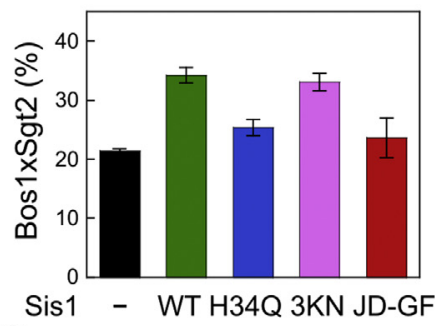

G

Bos $1^{\text {Bpa }}$ transfer from Ssa1-T201A to Sgt2

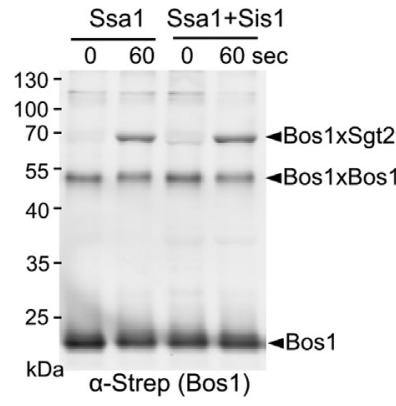

H

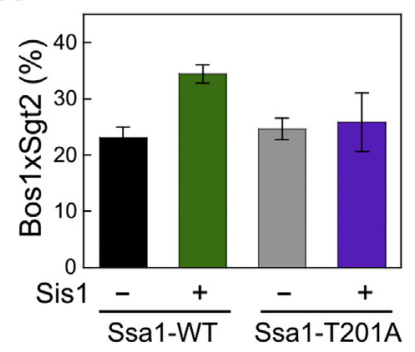

Figure 5. A functional J-domain in Ydj1 and Sis1 is required for enhancing the Ssa1-to-Sgt2 TA transfer. $A$ and $C$, representative Western blot images of Bos ${ }^{\text {Bpa }}$ transfer from Ssa1 to Sgt2 in the presence of the indicated Ydj1 $(A)$ or Sis1 $(C)$ variants. Reactions were carried out as described in Figure $3 A$. $B$ and $D$, quantification of the efficiency of the Bos1-Sgt2 crosslink from replicates of the data in $(A)$ and $(C)$, respectively-. $E$ and $G$, representative Western blot image of the time courses of Bos $1^{\mathrm{Bpa}}$ transfer from Ssa1(T201A) to Sgt2 in the absence and presence of Ydj1 $(E)$ or Sis1 $(G)$. $F$ and $H$, quantification of the efficiency of the Bos1-Sgt2 crosslink from replicates of the data in $(E)$ and $(G)$, respectively. All values in $(D),(F)$, and $(H)$ are reported as mean \pm SD, with $\mathrm{n} \geq$ 3. Error bars are shown but may not be visible in some cases. The lines in $(B)$ represent the mean from two independent measurements. TA, tail-anchored protein. 
wildtype Ydj1, whereas ATPase activation by Sis1(JD-GF) was comparable to Sis1(H34Q) (Fig. 4, $C-F$ ). These results suggest that Ydj1 JD-GF is necessary and sufficient for ATPase activation of Ssa1, whereas this activation requires participation by additional domains of Sis1. Mutation of three conserved lysines in Sis1-CTDI (K199/201/214N, or 3KN) disrupts the binding of CTDI with the EEVD motif of Ssa1 (44), but largely preserved the ATPase activation of Ssa1 (Fig. 4, $C-F$ ). These mutants allow us to uncouple the functions of JDPs in clientbinding and Hsp70 regulation. Finally, we introduced the T201A mutation in the Ssa1 ATPase site. Mutation of the homologous residue (T199A) in E. coli DnaK reduced the ATP hydrolysis rate without affecting ATP binding (45). We further confirmed that Ssa1(T201A) nearly abolished the Ydj1 and Sis1-mediated ATPase activation of Ssa1 (Fig. S7, $A$ and $B$ ). This mutant allowed us to assess whether ATPase activation on Ssa1 is required for the JDP-mediated regulations.

Using these mutants, we asked which domain(s) was used by Ydj1 to cooperate with Ssa1 during TA capture (Fig. 4, G and $H)$. In the absence of Ssa1, Ydj1(H34Q) modestly delayed the aggregation of Bos1, similar to observations with wildtype Ydj1, whereas Ydj1(JD-GF) had no effect on Bos1 aggregation, as expected from the lack of client binding domains (Fig. 4G, dotted lines). In the presence of equimolar Ssa1, Ydj1(H34Q) was less efficient than wildtype Ydj1 in helping to suppress the aggregation of Bos1 (Fig. 4G, solid lines and Fig. 4H). Ydj1 was also less efficient in assisting TA capture by mutant Ssa1(T201A) (Fig. 4, $I$ and $J$, also see Fig. 2, $A-C$ ), which abolishes the JDP-induced ATPase activation of Ssa1 (Fig. S7B), as does Ydj1(H34Q). In contrast, the T201A mutation did not affect the chaperone activity of Ssa1 toward the TA in the absence of Ydj1 (Fig. S8, $A$ and $B$ ). These observations indicate that the J-domain-mediated ATPase activation of Ssa1 plays an important role in the cooperation of Ydj1 with Ssa1 during TA capture. Ydj1(JD-GF) abolished the synergy with Ssa1 in preventing Bos1 aggregation (Fig. 4, $G$ and $H$, red), indicating that client binding by the Ydj1 CTDI/CTDII is required for Ydj1 to assist Ssa1 during TA trapping. Finally, none of the Sis1 variants assisted Ssa1 in TA trapping, as expected from the lack of this activity with wildtype Sis1 (Fig. S8, $C$ and $D$ ).

We further dissected the functional requirement of JDPs during the Ssa1-to-Sgt2 TA transfer. Despite its inability to bind Bos1 or help Ssa1 in TA capture (Fig. 4, $G$ and $H$ ), Ydj1(JD-GF) was sufficient to enhance the fraction of Bos1 crosslinked to Sgt2, with efficiencies comparable to that of Ydj1-WT (41.2 $\pm 1.7 \%$ and $39.9 \pm 2.3 \%$, respectively; Fig. 5, $A$ and $B$ ). In contrast, Ydj1(H34Q) did not alter the transfer efficiency of Bos1 (Fig. 5, $A$ and $B$ ). These results indicate that the interaction and regulation of Ssa1 by the Ydj1 Jdomain is necessary and sufficient for enhancing the Ssa1to-Sgt2 TA transfer. Both Sis1 mutants H34Q and JD-GF significantly reduced the Sis1-induced enhancement of TA transfer from Ssa1 to Sgt2, whereas mutant Sis1(3KN) stimulated the transfer almost as well as WT Sis1 (Fig. 5, C and $D$ ). Importantly, the enhancement of TA transfer efficiencies observed with the Ydj1 and Sis1 variants strongly correlated with their ability to activate ATP hydrolysis of Ssa1 (Fig. 4, B-F). Finally, Ssa1(T201A) abolished the ability of both JDPs to enhance the Bos1-Sgt2 crosslink after TA transfer (Fig. 5, $E-H$ ), although this mutation did not affect TA transfer in the absence of the JDPs (Fig. S5, $A-C$ ). This provides additional evidence that the JDP-stimulated ATP hydrolysis in Ssa1 is required for the enhancement of TA transfer. Together, these data show that both Ydj1 and Sis1 use their J-domains to regulate the nucleotide state and conformation of the Ssa1-Bos1 complex, thus enabling more efficient TA transfer to Sgt2. In contrast, the client binding domains of Ydj1 are dispensable for this process, providing further evidence that the enhancement of the Ssa1-to-Sgt2 TA transfer by Ydj1 can be uncoupled from its role in assisting Ssa1 in TA capture and preventing TA aggregation.

\section{A functional J-domain is required for JDPs to support efficient $T A$ insertion in vivo}

To test which domain(s) and activities of JDP are important for assisting TA insertion in vivo, we carried out complementation analyses. After depletion of both Sis1 and Ydj1 in the tetYDJ1/tet-SIS1 strain by doxycycline treatment as described earlier (25), we induced the expression of myctagged Ydj1, Sis1, or their variants and measured the ER insertion of BirA-Bos1 using pulse-chase assays. At 5 min after the chase, depletion of Ydj1 and Sis1 reduced the insertion of BirA-Bos1 from $\sim 72 \%$ to $27 \%$ (Fig. $6, A$ and $B$ ). Overexpression of WT Ydj1 rescued TA insertion to 47\%, whereas the $\mathrm{Ydj} 1(\mathrm{H} 34 \mathrm{Q})$ mutant provided no rescue compared with the empty vector (EV) control (Fig. 6, $A$ and $B$ ). Despite its inability to assist Ssa1 in TA capture (Fig. 2, $H$ and $I$ ) and its lower expression levels compared with Ydj1 (Fig. 6C), expression of Sis1 rescued TA insertion slightly more effectively than Ydj1 (Fig. 6, $A$ and $B$ ). Sis1(H34Q) reduced the rescue to levels comparable to the $\mathrm{EV}$ control, whereas Sis1(3KN) rescued the insertion of BirA-Bos1 as effectively as WT Sis1 (Fig. 6, $A$ and $B$ ). Anti-myc Western blot showed comparable expression levels for Sis1, Sis1(H34Q), and Sis1 $(3 \mathrm{KN})$ (Fig. 6C), indicating that the different rescues observed with WT and mutant Sis1 reflect differences in in vivo activity rather than their abundance. Although Ydj1(H34Q) was expressed at a lower level than WT Ydj1, the abundance of Ydj1(H34Q) was comparable to those of WT and mutant Sis1, suggesting that the failure of $\mathrm{Ydj} 1(\mathrm{H} 34 \mathrm{Q})$ to rescue the ER insertion of BirA-Bos1 was not because of insufficient protein expression levels. Together, these results show that a functional J-domain in Ydj1 and Sis1 to regulate Hsp70 activity is required for the ability of these JDPs to support efficient TA insertion.

\section{Discussion}

The effectiveness and versatility of Hsp70 function rely on its cooperation with JDPs, which drive diverse and specific functions of Hsp70s $(22,23,46)$. The results from this and a recent work (25) showed that among the 13 yeast cytosolic JDPs, two JDPs from distinct classes, Ydj1 and Sis1, serve 
A

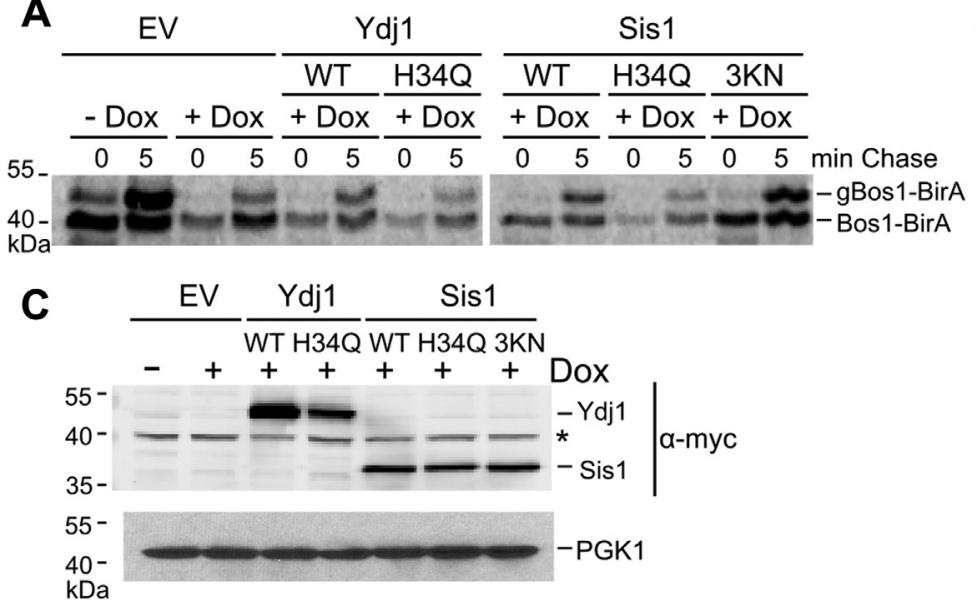

B

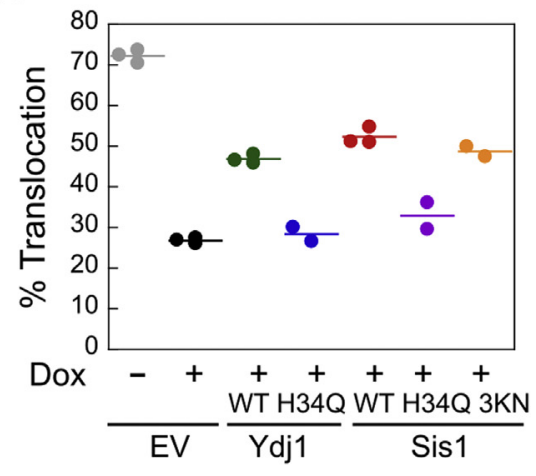

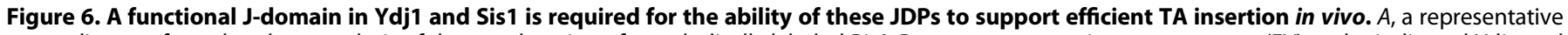

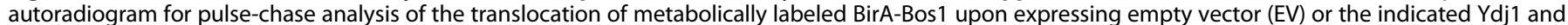

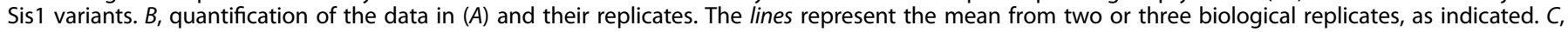

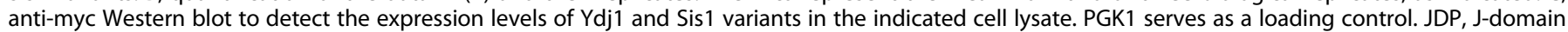
protein; TA, tail-anchored protein.

redundant roles in promoting the targeted delivery of multiple classes of membrane proteins, including the targeting of GETand GET-independent substrates to the ER (this work) and the import of $\beta$-barrel proteins to mitochondria (25). Further, biochemical analyses in the GET pathway uncovered a new activity of JDPs in enhancing the transfer competence of Hsp70-bound TAs to downstream chaperones. These findings provide evidence that in addition to their previously established roles in facilitating client capture on Hsp70s, JDPs enable Hsp70 to bind client proteins in a conformation more conducive to their successful biogenesis.

The ability of JDPs to assist in client trapping on Hsp70 is well studied $(38,39,47,48)$ and typically occurs via two mechanisms. First, the J-domain activates ATP hydrolysis on Hsp70, converting the latter to the ADP-bound state that binds substrates more tightly $(4,49,50)$. Second, some JDPs contain client-binding motifs that can also bind aggregationprone proteins $(38,39,47,48)$. Our results showed that Ydj1 uses both mechanisms to assist Ssa1 in capturing and maintaining the solubility of newly synthesized TAs. Nevertheless, this activity is not shared by Sis1, a class B JDP. These findings are consistent with the differences between class A and class B JDPs reported previously. Multiple peptide binding sites have been detected in Ydj1's CTDI, CTDII, and zinc-finger motif, which provide redundant interactions for client binding, whereas Sis1 and other type B JDPs contain only one peptide binding site in CTDI $(13,41,51)$. Moreover, peptide binding in Sis1 is displaced by the EEVD motif of Hsp70, whereas peptide interaction with Ydj1 persists in the presence of Hsp70 (41). These differences could explain why only Ydj1 synergizes with Ssa1 during TA capture, whereas Sis1 did not. Importantly, Sis1 can replace Ydj1 to support efficient TA insertion into the ER in vivo (Figs. 1 and 6) despite its inability to assist Ssa1 in TA trapping. This strongly suggests that the roles of JDPs in facilitating the ER-targeting of TAs cannot be solely attributed to enhanced TA trapping by Hsp70.
Unexpectedly, an activity shared between Ydj1 and Sis1 during their participation in the GET pathway is to improve the transfer of Ssa1-bound TA onto the downstream cochaperone Sgt2. In contrast to the initial TA trapping event that requires the client binding domain of Ydj1, the Ydj1 J-domain is necessary and sufficient to enhance this transfer. Moreover, Sis1 enhanced this transfer as efficiently as Ydj1 despite its inability to synergize with Ssa1 during initial TA capture. These observations exclude the possibility that this enhancement was because of better capture and solubilization of TA by the JDP/Hsp70 pair and suggest instead that JDP-induced changes in the conformation of the Ssa1.TA complex are responsible for the improvement in client transfer. The loss of this enhancement by the H34Q mutation in both JDPs or the T201A mutation in Ssa1 further indicates that the JDPinduced ATPase activation of Ssa1 is required for the enhanced TA transfer competence. While this observation is counter-intuitive, as Hsp70 binds client proteins more tightly in the ADP-state because of lid closing in the SBD (52-54), there is growing evidence that ADP-bound Hsp70 does not fully close the lid when it is loaded with protein substrates (5, $6,55)$. We propose that the Ydj1 and Sis1 J-domains regulate the conformation and nucleotide state of Ssa1, inducing it to bind TAs in a conformation that is more conducive to subsequent loading on Sgt2. These results extend the recent works showing that DnaJ/K can release client proteins in a more folding-competent conformation (15) and demonstrate that JDPs play an essential role in this client conformational regulation during the biogenesis of an essential class of integral membrane proteins. The enhanced folding competence of client proteins as a result of cooperation between JDP and Hsp70 could be envisioned in the biogenesis of a broad array of Hsp70 clients (32).

The roles of Ydj1/Sis1 in generating a more targetingcompetent conformation of the Ssa1.TA complex may not be limited to the GET pathway. Previous work suggested the 
presence of multiple redundant pathways in yeast that together form a robust network for the targeting of TAs and other membrane proteins to the ER $(34,36,56)$. Curiously, deletion of Sgt2 has a much weaker phenotype than the deletion of downstream genes including Get3 and Get1/2 (56-58), which was suggested to reflect the increased commitment of TA substrates to the GET pathway as they engage factors downstream of Sgt2. In support of this notion, Sbh1 forms cytosolic aggregates in $\Delta$ get 2 cells but is efficiently inserted into the ER membrane in $\Delta$ sgt $2 \Delta$ get 2 cells (56), indicating that TAs can be readily re-routed to alternative targeting pathways in the absence of Sgt2. Another puzzling observation is that transient inactivation of Ssa1 or depletion of Ydj1/Sis1 caused significantly larger defects in TA insertion than the deletion of Sgt2 (this work and (31)). This may be explained by the observation in this work that Ssa1 together with Ydj1/Sis1 are also required for the efficient in vivo targeting of GET-independent TAs as well as SND substrates. Collectively, these results suggest a model in which the cytosolic JDP/Hsp70 forms a chaperone hub upstream of multiple, redundant membrane protein insertion pathways (Fig. 7, step 7). The ability of Ydj1/Sis1 to generate a more targeting-competent conformation of TA on Ssa1 could contribute to enhanced TA insertion via the alternative pathways, and possibly also to the insertion of mitochondrial TAs as recently described (25).

We propose a new working model for the Hsp70/40mediated biogenesis of TAs in the GET pathway. The low intrinsic ATPase activity of Ssa1 (59) and the 10-fold excess of ATP over ADP in vivo maintain most of the free Hsp70s in the ATP-bound state. In this state, Ssa1 can rapidly capture newly synthesized TAs and protect them from irreversible aggregation in the aqueous cytosolic environment (Fig. 7, step 1 and dashed arrows). A class A JDP, Ydj1, can assist Hsp70 in this initial capture, although this activity is not shared by the class B JDP, Sis1, and does not appear to be required. Both Ydj1 and
Sis1 use their J-domains to activate ATP hydrolysis in Ssa1 and alter the conformation of the Ssa1.TA complex (Fig. 7, step 1-2). The Ssa1-TA complex recruits the cochaperone Sgt2 via interaction of the Ssa1 C-terminal motif with the Sgt2 TPR domain and transfers the bound TA to Sgt2 (Fig. 7, step 2-3). The JDP-induced changes in the conformation and nucleotide state of Ssa1 generate a more active transfer complex in which the TA is loaded onto Sgt2 with higher efficiency (Fig. 7, transfer complex in bracket). With the help of Get4/5, Sgt2 further hands off the TA substrate to the targeting factor Get3, thus committing the TA for delivery to the ER (Fig. 7, step 4-6). The JDP.Ssa1-TA complex could also be directed into alternative pathways if the TA fails to engage with Sgt2 (Fig. 7, step 7).

\section{Experimental procedures}

\section{Strains}

YTJ100 (YMK120a ydj1::tetO7-Ubiquitin-Leu-YDJ1(NatMX)), YTJ102 (YMK120a sis1::tetO7-Ubiquitin-Leu-SIS1(NatMX)), and YTJ138 (YMK120a sis1::tetO7-Ubiquitin-Leu-SIS1 (NatMX); ydj1::tetO7-Ubiquitin-Leu-YDJ1 (KanMX)) were kindly provided by Doron Rapaport (25). BY4741 (WT) and Aget3 (BY4741 YDL100C::KanMX) strains were purchased from American Type Culture Collection. SSA1 (MATa leu2-3112 his3-11 ura3-52

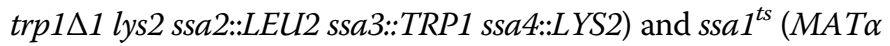
leu2-3112 his3-11 ura3-52 trp1A1 lys2 ssa1-45:URA3 ssa2::LEU2 ssa3::TRP1 ssa4::LYS2) strains were kindly provided by Dr Elizabeth A. Craig (37).

\section{Protein expression and purification}

Strep-SUMO-Bos1 and $\mathrm{His}_{6}$-Sgt2 were expressed and purified as described previously $(31,35)$.

To express Strep-SUMO-Bos $1^{\mathrm{Bpa}}$, the coding sequence for the eighth amino acid residue (Ile) in the Bos1-TMD
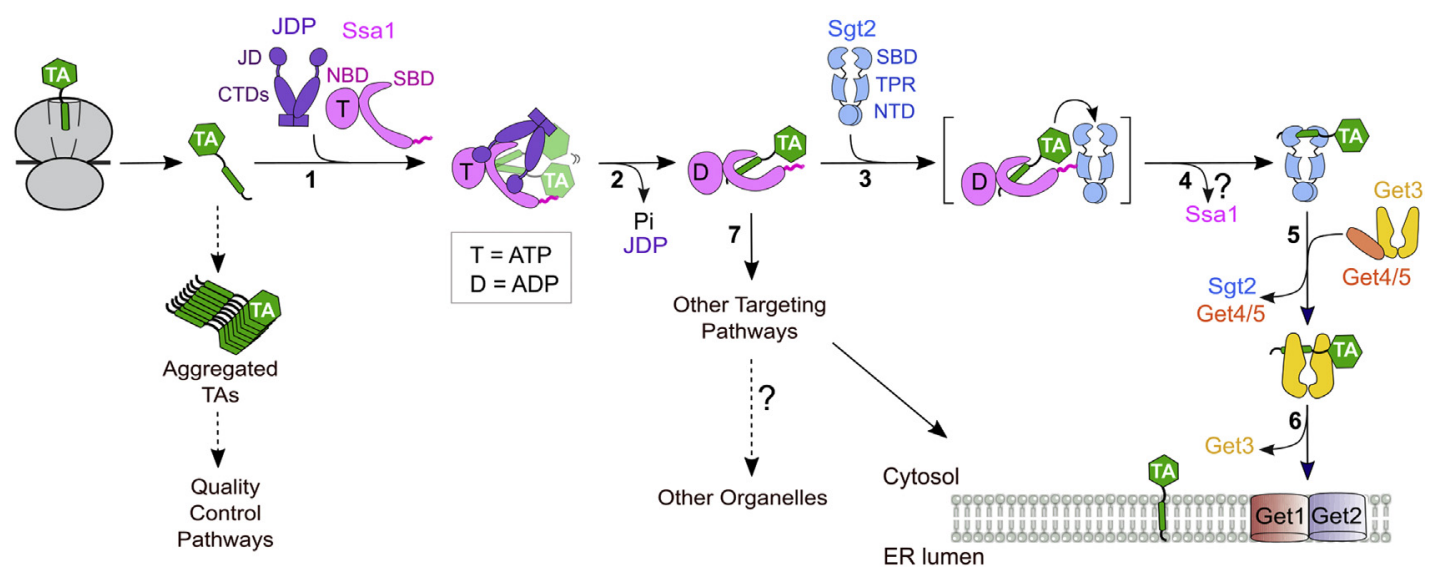

Figure 7. Revised model of TA targeting by the GET pathway in yeast. Step 1 to 2, TAs released from the ribosome are captured by Ssa1 and protected from aggregation in the cytosol (dashed arrows). The efficiency of this step can be further enhanced by Ydj1 but not Sis1. The J-domain of Ydj1 or Sis1 stimulates ATP hydrolysis on Ssa1 and drives it to bind TA in an altered conformation. Step 3, the TA transfer complex is assembled via association of Ssa1 with the Sgt2 TPR domain. The Ydj1- or Sis1-induced conformational changes in Ssa1 also enhance the transfer competence of the TA. Step 4, TA is transferred from Ssa1 to Sgt2. The question mark indicates that it is unclear whether Ssa1 dissociates from Sgt2 after TA transfer. Step 5, TAs undergo a second transfer from Sgt2 to Get3 assisted by the Get4/5 complex. Step 6, Get3 delivers TAs to the Get1/2 receptors for insertion into the ER membrane. Step 7, TAs bound to JDP.Ssa1 could enter alternative targeting pathways to be targeted to the ER or to other organelles. JDP, J-domain protein; TA, tailanchored protein. 
(LVFWIALILLIIGIYYVL) was replaced by an amber codon (TAG) using the expression plasmid for Strep-SUMO-Bos1 (31) as a template and QuikChange mutagenesis (Agilent Technologies, Inc.). Expression plasmids for Strep-SUMOBos1 $^{\mathrm{Amb}}$ and tRNA $_{\mathrm{CUA}}{ }^{\mathrm{Opt}}$ synthetase (43) were cotransformed into BL21 Star (DE3) cells (Thermo Fisher Scientific Inc.). The expression of RNA $_{C U A}{ }^{\text {Opt }}$ synthetase was induced at $\mathrm{OD}_{600}$ of 0.4 using $0.2 \%$ arabinose and then $1 \mathrm{mM}$ Bpa was supplemented in the media. At $\mathrm{OD}_{600}$ of 0.6 , expression of Strep-SUMO-Bos $1^{\text {Amb }}$ was induced with $0.1 \mathrm{mM}$ isopropyl $\beta$-D-1-thiogalactopyranoside for $100 \mathrm{~min}$ at $37{ }^{\circ} \mathrm{C}$. Bpa incorporation into Bos1 was confirmed by SDSPAGE analysis. The purification of Strep-SUMO-Bos $1^{\text {Bpa }}$ was performed as described previously, and then, the purified protein was stored in buffer A (50 mM Hepes [pH 7.5], $150 \mathrm{mM} \mathrm{NaCl}, 10 \%$ glycerol, 0.05\% LDAO) (31).

$\mathrm{His}_{6}$-SUMO-Ssa1, His ${ }_{6}$-SUMO-Ssa1-T201A, His 6 -SUMOYdj1, His $_{6}$-SUMO-Ydj1-H34Q, His $_{6}$-SUMO-Ydj1-JDGF(1-102aa), His $_{6}$-SUMO-Sis1, His ${ }_{6}$-SUMO-Sis1-H34Q, $\mathrm{His}_{6}$-SUMO-Sis1-K199N/K202N/K214N (3KN), and $\mathrm{His}_{6}{ }^{-}$ where $\mathrm{S}_{\mathrm{Max}}$ is the \% soluble TA at saturating Ssa1 concentrations, and $K_{\text {soluble }}$ is the apparent TA binding constant.

\section{Sedimentation analysis of recombinant Bos 1}

$90 \mu \mathrm{M}$ Strep-SUMO-Bos1 stored in buffer A was diluted to a final concentration of $3 \mu \mathrm{M}$ in assay buffer containing indicated concentrations of chaperones and incubated at RT for $5 \mathrm{~min}$. The reactions were ultracentrifuged at $100,000 \mathrm{rpm}$ for $30 \mathrm{~min}$ using TLA 100 rotor (Beckman Coulter). Total input $(\mathrm{T})$, soluble $(\mathrm{S})$, and pellet $(\mathrm{P})$ fractions were resolved by SDS-PAGE and detected by Western blot using the following antibodies: Bos1, anti-Strep; Sgt2 or Ydj1, anti-His; Ssa1, anti-Ssa (a gift from Elizabeth A. Craig). Western blots were imaged using Odyssey Imager (LI-COR Inc.) and quantified using Quantity One software (Bio-Rad). The concentration of soluble TA $\left(S_{o b s d}\right)$ was calculated as [Total Bos1 concentration" $\mathrm{I}_{\mathrm{S}} / \mathrm{I}_{\mathrm{T}}$ ], where $\mathrm{I}_{\mathrm{S}}$ and $\mathrm{I}_{\mathrm{T}}$ denote the intensities of the bands of $\mathrm{S}$ and $\mathrm{T}$ fractions, respectively. The Ssa1 concentration dependence of $S_{\text {obsd }}$ values was fit to Equation 2,

$$
S_{\text {obsd }}=S_{\text {Max }} \times\left\{\frac{[T A]+[\text { Ssa } 1]+K_{\text {Soluble }}-\sqrt{\left([T A]+[\text { Ssa } 1]+K_{\text {Soluble }}\right)^{2}-4[T A][\text { Ssa } 1]}}{2[T A]}\right\}
$$

SUMO-Sis1-JD-GF(1-121aa) were expressed and purified as previously described (31) with minor modifications. After protein expression, cells were resuspended in buffer B $(20 \mathrm{mM}$ Tris [pH 8.0], $500 \mathrm{mM} \mathrm{NaCl}, 10 \%$ glycerol, $2 \mathrm{mM} \beta$-ME, $15 \mathrm{mM}$ imidazole) supplemented with protease inhibitor cocktail. Clarified lysate was incubated with Ni Sepharose High Performance resin (GE Healthcare). The column was washed with buffer B and eluted with buffer B containing $300 \mathrm{mM}$ imidazole. To obtain tagless Ssa1, Ydj1, and their mutants, purified $\mathrm{His}_{6}$-SUMO fusion proteins were digested with SUMO protease overnight at $4{ }^{\circ} \mathrm{C}$, and the proteins were further purified using either MonoQ 5/50GL or MonoS 5/ 50GL (GE Healthcare).

\section{Turbidity assay}

$90 \mu \mathrm{M}$ Strep-SUMO-Bos1 stored in buffer A was rapidly (within $15 \mathrm{~s}$ ) diluted to a final concentration of $1.5 \mu \mathrm{M}$ in assay buffer $(20 \mathrm{mM}$ K-Hepes [pH 7.5], $150 \mathrm{mM}$ KOAc, $5 \mathrm{mM} \mathrm{Mg}(\mathrm{OAc})_{2}, 2 \mathrm{mM} \beta$-ME, $\left.2 \mathrm{mM} \mathrm{ATP}\right)$ containing indicated concentrations of chaperones (Ssa1 or/and Ydj1). The optical density at $360 \mathrm{~nm}$ was measured in real time using Spectrophotometer DU 640 (Beckman Coulter). The observed solubility of Bos1 $\left(\mathrm{S}_{\text {obsd }}\right.$ ) was calculated from the \% change of optimal readings at 5 min between Bos1 alone and chaperonecontaining samples. The data were plotted as a function of Ssal concentration and fit to Equation 1,

$$
S_{\text {obsd }}=S_{\text {Max }} \times \frac{[\text { Ssa } 1]}{K_{\text {soluble }}+[S s a 1]}
$$

in which $\mathrm{S}_{\mathrm{Max}}$ is the concentration of Bos1 at saturating Ssa1 concentrations, and $K_{\text {Soluble }}$ is the apparent binding constant of Ssa1 or Ssa1·Ydj1 to Bos1.

\section{TA transfer reaction from Ssa1 to Sgt2}

$80 \mu \mathrm{M}$ Strep-SUMO-Bos $1^{\mathrm{Bpa}}$ stored in buffer A was diluted to a final concentration of $0.1 \mu \mathrm{M}$ in assay buffer containing $3 \mu \mathrm{M}$ Ssa 1 or/and $3 \mu \mathrm{M}$ Ydj1 and incubated for $1 \mathrm{~min}$ at room temperature. $\mathrm{His}_{6}-\mathrm{Sgt} 2$ was added to a final concentration of $0.3 \mu \mathrm{M}$ in the reaction $(100 \mu \mathrm{l})$, and reactions were further incubated at room temperature. At indicated time points, $10 \mu \mathrm{l}$ aliquots were removed from the reaction and quenched by flash freezing in liquid nitrogen. $0 \mathrm{~s}$ samples were taken right before Sgt2 was added in the reactions. Frozen aliquots were subsequently crosslinked on dry ice $\sim 4 \mathrm{~cm}$ away from a UVP B-100AP lamp (UVP LLC) for 90 min. Crosslinked bands were resolved on SDS-PAGE and analyzed by Western blot. Crosslinking efficiency was calculated as $\left[\mathrm{I}_{\text {Bos1xSgt2 }} /\left(\mathrm{I}_{\text {Bos1 }}+\right.\right.$ $\left.\left.\mathrm{I}_{\text {Bos1xSgt2 }}+\mathrm{I}_{\text {Bos1xYdj1 }}\right]\right]^{*} 100$, where I denotes the intensity of the band of interest.

\section{Single turn-over ATPase assay}

$3 \mu \mathrm{M}$ Ssa1 with or without $3 \mu \mathrm{M}$ Ydj1 variants in reaction buffer $(20 \mathrm{mM}$ K-Hepes [pH 7.5], $150 \mathrm{mM}$ KOAc, $5 \mathrm{mM}$ $\mathrm{Mg}(\mathrm{OAc})_{2}, 5 \mathrm{mM} \beta$-ME $)$ was mixed with $2.4 \mu \mathrm{M}$ ATP containing $\gamma^{32} \mathrm{P}$-ATP and incubated at room temperature. At indicated time points, $2 \mu \mathrm{l}$ aliquots were removed from the reaction and quenched in $4 \mu \mathrm{l}$ TLC solution $(500 \mathrm{mM} \mathrm{LiCl}, 1$ 
M Formic acid). $\gamma^{32} \mathrm{P}$-ATP and $\gamma^{32} \mathrm{P}$-Pi were separated by thin layer chromatography using PEI cellulose (Vendor) and quantified by phosphorimaging using a Typhoon imager (GE Healthcare). Time courses of the reaction were fit to Equation 3,

$$
[A T P]_{t}=a \times e^{-k_{o b s} t}
$$

in which $a$ is the fraction of ATP before initiation of the reaction, and $k_{o b s d}$ is the observed rate constant for Ssa1 ATP hydrolysis.

\section{Pulse-chase experiments}

Plasmids (pRS313-BirA-Bos1, pRS313-Bos1-BirA, pRS313DHC- $\alpha$ F, pRS313-BirA-6AG, and pRS313-Scs2-GFP) were transformed into indicated yeast cells. Colonies were grown in selective media (SD-His) until $\mathrm{OD}_{600}$ reached 0.2. Cells were supplemented with $2 \mu \mathrm{g} / \mathrm{ml}$ doxycycline (Sigma-Aldrich) and cultured for an additional $4 \mathrm{~h}$ at $30^{\circ} \mathrm{C}$. Cells were then washed with water and resuspended in SD-His-Met media at $8 \mathrm{OD}_{600}$ units per ml. After growing at $30{ }^{\circ} \mathrm{C}$ for $30 \mathrm{~min}$, cells were pulse-labeled with $200 \mu \mathrm{Ci} / \mathrm{ml}$ EasyTagTM EXPRESS35S Protein Labeling Mix (PerkinElmer) for 2 min and chased with $10 \mathrm{mM}$ cold methionine. Cells $(200 \mu \mathrm{l})$ were removed from the culture and flash-frozen in liquid nitrogen at indicated times during the chase.

Pulse-chase experiments with BirA-6AG and Scs2-GFP in $S S A 1$ and $s s a 1^{t}$ cells were performed as previously described (31).

For complementation assays, Ydj1 or Sis1 variants (pESCTrp-Myc-Ydj1, pESC-Trp-Myc-Ydj1-H34Q, pESC-Trp-MycSis1, pESC-Trp-Myc-Sis1-H34Q, and pESC-Trp-Myc-Sis1$3 \mathrm{KN})$ were co-transformed with pRS313-BirA-Bos1 into YTJ138 (YMK120a sis1::tetO7-Ubiquitin-Leu-SIS1 (NatMX); ydj1::tetO7-Ubiquitin-Leu-YDJ1 (KanMX)) cells. Colonies were grown in SD-His-Trp media overnight, and cells were washed and resuspended in selective media containing $2 \%$ Raffinose and $0.05 \%$ Glucose. After adjusting $\mathrm{OD}_{600}$ to $\sim 0.2$, cells were cultured for $4 \mathrm{~h}$ at $30^{\circ} \mathrm{C}$, and $2 \%$ galactose and $2 \mu \mathrm{g} /$ $\mathrm{ml}$ doxycycline were supplemented. Cells were further cultured for $4 \mathrm{~h}$ at $30{ }^{\circ} \mathrm{C}$, and pulse-chase experiments were performed as described above.

The frozen cell samples were lysed and immunoprecipitated using anti-HA magnetic beads as previously described (31). Immunoprecipitated samples were resolved by SDS-PAGE and quantified by autoradiography. Translocation efficiency

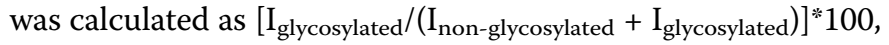
where I denotes the intensity of the band of interest.

\section{Quantification and statistical analysis}

Statistical details of each experiment can be found in the figure legends. For both Western blot and radiographic images, Quantity One 1-D Analysis Software (Bio-Rad) was used to quantify the intensities of bands. The value of $n$ is specified in each figure legend, as well as the $p$ value when statistical test was performed.

\section{Data availability}

All data described are contained in the manuscript.

Supporting information-This article contains supporting information.

Acknowledgments-We thank D. Rapaport, T. Jores for the yeast strains, E. A. Craig for yeast strains and for antibodies against Ssa and Sis1, the Center for Molecular Medicine (California Institute of Technology) for use of the Li-Cor Odyssey imager, and members of the Shan laboratories for critical comments on the manuscript.

Author contributions-H. C. and S. S. designed experiments; H. C., W. J. S., and Y. L. performed experiments and analyzed the data; H. C. and S. S. wrote the paper. All authors reviewed the results and approved the final version of the manuscript.

Funding and additional information-This work was supported by NIH grant R01 GM107368 and R35 GM136321 to S. S. The content is solely the responsibility of the authors and does not necessarily represent the official views of the National Institutes of Health.

Conflict of interest-The authors declare that they have no conflicts of interest with the contents of this article.

Abbreviations-The abbreviations used are: CTD, C-terminal domain; EV, empty vector; GET, guided entry of tail-anchored protein; JDP, J-domain protein; NBD, nucleotide binding domain; SND, SRP-independent; SBD, substrate binding domain; SRP, signal recognition particle; TMD, transmembrane domain; TPR, tetratricopeptide repeat.

\section{References}

1. Clerico, E. M., Tilitsky, J. M., Meng, W., and Gierasch, L. M. (2015) How hsp70 molecular machines interact with their substrates to mediate diverse physiological functions. J. Mol. Biol. 427, 1575-1588

2. Mashaghi, A., Bezrukavnikov, S., Minde, D. P., Wentink, A. S., Kityk, R., Zachmann-Brand, B., Mayer, M. P., Kramer, G., Bukau, B., and Tans, S. J. (2016) Alternative modes of client binding enable functional plasticity of Hsp70. Nature 539, 448-451

3. Mayer, M. P., and Bukau, B. (2005) Hsp70 chaperones: Cellular functions and molecular mechanism. Cell Mol. Life Sci. 62, 670-684.

4. Schmid, D., Baici, A., Gehring, H., and Christen, P. (1994) Kinetics of molecular chaperone action. Science 263, 971-973

5. Marcinowski, M., Höller, M., Feige, M. J., Baerend, D., Lamb, D. C., and Buchner, J. (2011) Substrate discrimination of the chaperone BiP by autonomous and cochaperone-regulated conformational transitions. Nat. Struct. Mol. Biol. 18, 150-158

6. Schlecht, R., Erbse, A. H., Bukau, B., and Mayer, M. P. (2011) Mechanics of Hsp70 chaperones enables differential interaction with client proteins. Nat. Struct. Mol. Biol. 18, 345-351

7. Dragovic, Z., Broadley, S. A., Shomura, Y., Bracher, A., and Hartl, F. U. (2006) Molecular chaperones of the Hsp110 family act as nucleotide exchange factors of Hsp70s. EMBO J. 25, 2519-2528

8. Gowda, N. K. C., Kaimal, J. M., Kityk, R., Daniel, C., Liebau, J., Öhman, M., Mayer, M. P., and Andréasson, C. (2018) Nucleotide exchange factors 
Fes1 and HspBP1 mimic substrate to release misfolded proteins from Hsp70. Nat. Struct. Mol. Biol. 25, 83-89

9. Raviol, H., Sadlish, H., Rodriguez, F., Mayer, M. P., and Bukau, B. (2006) Chaperone network in the yeast cytosol: Hsp110 is revealed as an Hsp70 nucleotide exchange factor. EMBO J. 25, 2510-2518

10. Kityk, R., Kopp, J., and Mayer, M. P. (2018) Molecular mechanism of Jdomain-triggered ATP hydrolysis by Hsp70 chaperones. Mol. Cell. 69, 227-237.e4

11. Tsai, J., and Douglas, M. G. (1996) A conserved HPD sequence of the Jdomain is necessary for YDI1 stimulation of Hsp70 ATPase activity at a site distinct from substrate binding. J. Biol. Chem. 271, 9347-9354

12. Wall, D., Zylicz, M., and Georgopoulos, C. (1994) The NH2-terminal 108 amino acids of the Escherichia coli DnaJ protein stimulate the ATPase activity of DnaK and are sufficient for lambda replication. J. Biol. Chem. 269, 5446-5451

13. Fan, C.-Y., Ren, H.-Y., Lee, P., Caplan, A. J., and Cyr, D. M. (2005) The type I Hsp40 zinc finger-like region is required for Hsp70 to capture nonnative polypeptides from Ydj1. J. Biol. Chem. 280, 695-702

14. Li, J., Qian, X., and Sha, B. (2003) The crystal structure of the yeast Hsp40 Ydj1 complexed with its peptide substrate. Structure 11, 1475-1483

15. Imamoglu, R., Balchin, D., Hayer-Hartl, M., and Hartl, F. U. (2020) Bacterial Hsp70 resolves misfolded states and accelerates productive folding of a multi-domain protein. Nat. Commun. 11, 365

16. Kampinga, H. H., and Craig, E. A. (2010) The HSP70 chaperone machinery: J proteins as drivers of functional specificity. Nat. Rev. Mol. Cell Biol. 11, 579-592

17. Kampinga, H. H., Andreasson, C., Barducci, A., Cheetham, M. E., Cyr, D., Emanuelsson, C., Genevaux, P., Gestwicki, J. E., Goloubinoff, P., HuertaCepas, J., Kirstein, J., Liberek, K., Mayer, M. P., Nagata, K., Nillegoda, N. B., et al. (2019) Function, evolution, and structure of J-domain proteins. Cell Stress Chaperones 24, 7-15

18. Sahi, C., and Craig, E. A. (2007) Network of general and specialty J protein chaperones of the yeast cytosol. Proc. Natl. Acad. Sci. U. S. A. 104, 71637168

19. Krantz, K. C., Puchalla, J., Thapa, R., Kobayashi, C., Bisher, M., Viehweg, J., Carr, C. M., and Rye, H. S. (2013) Clathrin coat disassembly by the yeast $\mathrm{Hsc70/Ssa1p}$ and auxilin/Swa2p proteins observed by single-particle burst analysis spectroscopy. J. Biol. Chem. 288, 26721-26730

20. Sahi, C., Lee, T., Inada, M., Pleiss, J. A., and Craig, E. A. (2010) Cwc23, an essential J protein critical for pre-mRNA splicing with a dispensable J domain. Mol. Cell. Biol. 30, 33-42

21. Weyer, F. A., Gumiero, A., Gesé, G. V., Lapouge, K., and Sinning, I. (2017) Structural insights into a unique Hsp70-Hsp40 interaction in the eukaryotic ribosome-associated complex. Nat. Struct. Mol. Biol. 24, 144151

22. Becker, T., Song, J., and Pfanner, N. (2019) Versatility of preprotein transfer from the cytosol to mitochondria. Trends Cell Biol. 29, 534-548

23. Craig, E. A. (2018) Hsp70 at the membrane: Driving protein translocation. BMC Biol. 16, 11

24. Caplan, A. J., Cyr, D. M., and Douglas, M. G. (1992) YDJ1p facilitates polypeptide translocation across different intracellular membranes by a conserved mechanism. Cell 71, 1143-1155

25. Jores, T., Lawatscheck, J., Beke, V., Franz-Wachtel, M., Yunoki, K., Fitzgerald, J. C., Macek, B., Endo, T., Kalbacher, H., Buchner, J., and Rapaport, D. (2018) Cytosolic Hsp70 and Hsp40 chaperones enable the biogenesis of mitochondrial $\beta$-barrel proteins. J. Cell Biol. 217, 30913108

26. Ast, T., Cohen, G., and Schuldiner, M. (2013) A network of cytosolic factors targets SRP-independent proteins to the endoplasmic reticulum. Cell 152, 1134-1145

27. Opaliński, Ł., Song, J., Priesnitz, C., Wenz, L.-S., Oeljeklaus, S., Warscheid, B., Pfanner, N., and Becker, T. (2018) Recruitment of cytosolic Jproteins by TOM receptors promotes mitochondrial protein biogenesis. Cell Rep. 25, 2036-2043.e5

28. Papić, D., Elbaz-Alon, Y., Koerdt, S. N., Leopold, K., Worm, D., Jung, M., Schuldiner, M., and Rapaport, D. (2013) The role of Djp1 in import of the mitochondrial protein Mim1 demonstrates specificity between a cochaperone and its substrate protein. Mol. Cell. Biol. 33, 4083-4094.
29. Chio, U. S., Cho, H., and Shan, S.-O. (2017) Mechanisms of tail-anchored membrane protein targeting and insertion. Annu. Rev. Cell Dev. Biol. 33, $417-438$

30. Hegde, R. S., and Keenan, R. J. (2011) Tail-anchored membrane protein insertion into the endoplasmic reticulum. Nat. Rev. Mol. Cell Biol. 12, 787-798

31. Cho, H., and Shan, S.-O. (2018) Substrate relay in an Hsp70-cochaperone cascade safeguards tail-anchored membrane protein targeting. EMBO J. 37, e99264.

32. Ng, D. T., Brown, J. D., and Walter, P. (1996) Signal sequences specify the targeting route to the endoplasmic reticulum membrane. J. Cell Biol. 134, 269-278

33. Borgese, N., Coy-Vergara, J., Colombo, S. F., and Schwappach, B. (2019) The ways of tails: The GET pathway and more. Protein J. 38, 289-305

34. Aviram, N., Ast, T., Costa, E. A., Arakel, E. C., Chuartzman, S. G., Jan, C. H., Haßdenteufel, S., Dudek, J., Jung, M., Schorr, S., Zimmermann, R., Schwappach, B., Weissman, J. S., and Schuldiner, M. (2016) The SND proteins constitute an alternative targeting route to the endoplasmic reticulum. Nature 540, 134-138

35. Rao, M., Okreglak, V., Chio, U. S., Cho, H., Walter, P., and Shan, S.-O. (2016) Multiple selection filters ensure accurate tail-anchored membrane protein targeting. Elife 5, e21301

36. Shurtleff, M. J., Itzhak, D. N., Hussmann, J. A., Schirle Oakdale, N. T., Costa, E. A., Jonikas, M., Weibezahn, J., Popova, K. D., Jan, C. H., Sinitcyn, P., Vembar, S. S., Hernandez, H., Cox, J., Burlingame, A. L., Brodsky, J. L., et al. (2018) The ER membrane protein complex interacts cotranslationally to enable biogenesis of multipass membrane proteins. Elife 7, e37018

37. Becker, J., Walter, W., Yan, W., and Craig, E. A. (1996) Functional interaction of cytosolic hsp70 and a DnaJ-related protein, Ydj1p, in protein translocation in vivo. Mol. Cell Biol. 16, 4378-4386

38. Cyr, D. M. (1995) Cooperation of the molecular chaperone Ydj1 with specific Hsp70 homologs to suppress protein aggregation. FEBS Lett. 359, 129-132

39. Lu, Z., and Cyr, D. M. (1998) The conserved carboxyl terminus and zinc finger-like domain of the co-chaperone Ydj1 assist Hsp70 in protein folding. J. Biol. Chem. 273, 5970-5978

40. Wang, F., Brown, E. C., Mak, G., Zhuang, J., and Denic, V. (2010) A chaperone cascade sorts proteins for posttranslational membrane insertion into the endoplasmic reticulum. Mol. Cell. 40, 159-171

41. Jiang, Y., Rossi, P., and Kalodimos, C. G. (2019) Structural basis for client recognition and activity of Hsp40 chaperones. Science 365, 1313-1319

42. Lu, Z., and Cyr, D. M. (1998) Protein folding activity of Hsp70 is modified differentially by the hsp40 co-chaperones Sis1 and Ydj1. J. Biol. Chem. 273, 27824-27830

43. Young, T. S., Ahmad, I., Yin, J. A., and Schultz, P. G. (2010) An enhanced system for unnatural amino acid mutagenesis in E. coli. J. Mol. Biol. 395, 361-374

44. Yu, H. Y., Ziegelhoffer, T., Osipiuk, J., Ciesielski, S. J., Baranowski, M., Zhou, M., Joachimiak, A., and Craig, E. A. (2015) Roles of intramolecular and intermolecular interactions in functional regulation of the Hsp70 Jprotein co-chaperone Sis1. J. Mol. Biol. 427, 1632-1643

45. Barthel, T. K., Zhang, J., and Walker, G. C. (2001) ATPase-defective derivatives of Escherichia coli DnaK that behave differently with respect to ATP-induced conformational change and peptide release. J. Bacteriol. $183,5482-5490$

46. Craig, E. A., and Marszalek, J. (2017) How do J-proteins get Hsp70 to do so many different things? Trends Biochem. Sci. 42, 355-368

47. Aprile, F. A., Källstig, E., Limorenko, G., Vendruscolo, M., Ron, D., and Hansen, C. (2017) The molecular chaperones DNAJB6 and Hsp70 cooperate to suppress $\alpha$-synuclein aggregation. Sci. Rep. 7, 9039

48. Shorter, J., and Lindquist, S. (2008) Hsp104, Hsp70 and Hsp40 interplay regulates formation, growth and elimination of Sup35 prions. EMBO J. 27, 2712-2724

49. Mayer, M. P., Schröder, H., Rüdiger, S., Paal, K., Laufen, T., and Bukau, B. (2000) Multistep mechanism of substrate binding determines chaperone activity of Hsp70. Nat. Struct. Biol. 7, 586-593

50. Vogel, M., Bukau, B., and Mayer, M. P. (2006) Allosteric regulation of Hsp70 chaperones by a proline switch. Mol. Cell. 21, 359-367 
51. Kota, P., Summers, D. W., Ren, H.-Y., Cyr, D. M., and Dokholyan, N. V. (2009) Identification of a consensus motif in substrates bound by a Type I Hsp40. Proc. Natl. Acad. Sci. U. S. A. 106, 11073-11078

52. Banerjee, R., Jayaraj, G. G., Peter, J. J., Kumar, V., and Mapa, K. (2016) Monitoring conformational heterogeneity of the lid of DnaK substratebinding domain during its chaperone cycle. FEBS J. 283, 2853-2868

53. Mapa, K., Sikor, M., Kudryavtsev, V., Waegemann, K., Kalinin, S., Seidel, C. A. M., Neupert, W., Lamb, D. C., and Mokranjac, D. (2010) The conformational dynamics of the mitochondrial Hsp70 chaperone. Mol. Cell 38, 89-100

54. Wu, S., Hong, L., Wang, Y., Yu, J., Yang, J., Yang, J., Zhang, H., and Perrett, S. (2020) Kinetics of the conformational cycle of Hsp70 reveals the importance of the dynamic and heterogeneous nature of Hsp70 for its function. Proc. Natl. Acad. Sci. U. S. A. 117, 7814-7823

55. Lai, A. L., Clerico, E. M., Blackburn, M. E., Patel, N. A., Robinson, C. V., Borbat, P. P., Freed, J. H., and Gierasch, L. M. (2017) Key features of an
Hsp70 chaperone allosteric landscape revealed by ion-mobility native mass spectrometry and double electron-electron resonance. J. Biol. Chem. $292,8773-8785$

56. Kiktev, D. A., Patterson, J. C., Müller, S., Bariar, B., Pan, T., and Chernoff, Y. O. (2012) Regulation of chaperone effects on a yeast prion by cochaperone Sgt2. Mol. Cell. Biol. 32, 4960-4.970

57. Kohl, C., Tessarz, P., von der Malsburg, K., Zahn, R., Bukau, B., and Mogk, A. (2011) Cooperative and independent activities of Sgt2 and Get5 in the targeting of tail-anchored proteins. Biol. Chem. 392, 601-608

58. Powis, K., Schrul, B., Tienson, H., Gostimskaya, I., Breker, M., High, S., Schuldiner, M., Jakob, U., and Schwappach, B. (2013) Get3 is a holdase chaperone and moves to deposition sites for aggregated proteins when membrane targeting is blocked. J. Cell Sci. 126, 473-483

59. Ziegelhoffer, T., Lopez-Buesa, P., and Craig, E. A. (1995) The dissociation of ATP from hsp70 of Saccharomyces cerevisiae is stimulated by both Ydj1p and peptide substrates. J. Biol. Chem. 270, 10412-10419

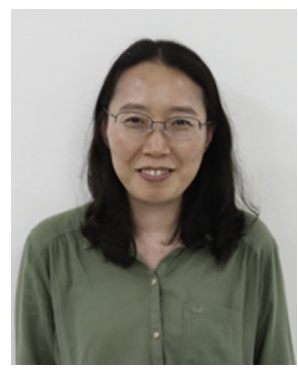

Hyunju Cho is a Young Scientist Fellow at the Institute for Basic Science, South Korea. During her PhD program at the Michigan State University, she studied molecular mechanisms by which a saturated fatty acid, palmitic acid, induces ER stressmediated apoptosis. While working as a postdoctoral researcher in the Shan laboratory at the California Institute of Technology, her works uncovered the functional roles of yeast Hsp70 and Hsp40 proteins in the guided-entry-of-tail-anchored protein pathway. She currently leads her independent research studying the targeting of other organellar membrane proteins and their therapeutic applications in protein homeostasis diseases. 